\title{
Linear Kondo conductance in a quantum dot
}

\author{
Domenico Giuliano $^{\ddagger}$, Adele Naddeo ${ }^{\dagger *}$ and Arturo Tagliacozzo ${ }^{\dagger *}$ \\ ¥ Dipartimento di Fisica, Università della Calabria, Arcavacata di Rende - Cosenza, Italy \\ $\dagger$ Coherentia - INFM (Istituto Nazionale di Fisica della Materia ), Unità di Napoli, Napoli, Italy \\ * Dipartimento di Scienze Fisiche Università di Napoli "Federico II ", \\ Monte S.Angelo - via Cintia, I-80126 Napoli, Italy
}

\begin{abstract}
In a tunneling experiment across a quantum dot it is possible to change the coupling between the dot and the contacts at will, by properly tuning the transparency of the barriers and the temperature. Gate voltages allow for changes of the relative position of the dot addition energies and the Fermi level of the leads. Here we discuss the two limiting cases: weak and strong coupling in the tunneling Hamiltonian. In the latter case Kondo resonant conductance can emerge at low temperature in a Coulomb blockade valley. We give a pedagogical approach to the single channel Kondo physics at equilibrium and review the Nozières scattering picture of the correlated fixed point. We emphasize the effect of an applied magnetic field and show how an orbital Kondo effect can take place in vertical quantum dots tuned both to an even and an odd number of electrons at a level crossing. We extend the approach to the two channel overscreened Kondo case and discuss recent proposals for detecting the non Fermi Liquid fixed point which could be reached at strong coupling.
\end{abstract}

\section{INTRODUCTION}

Recently systems have been fabricated which can sustain quantum coherence because of the smallness of their size, provided that the temperature is low enough. These mesoscopic systems are nanostructured devices in which quantum coherence sets in at very low temperatures and modifies the properties of the device as a whole. This happens notwithstanding the fact that the system is connected to an external biasing circuit. They have global, geometry dependent properties and striking quantization phenomena arise which are largely independent of the specific sample involved: charge quantization (in unit of the electron charge $e$ ), periodicity in the magnetic flux quantum $\phi_{o}=h c / e$, conductance quantization (in units of $G_{K}=2 e^{2} / \pi \hbar=(6.5 K \Omega)^{-1}$ ).

Among these macroscopic quantum phenomena there is the unitary limit of the Kondo conductance in tunneling across a quantum dot (QD) at Coulomb Blockade (CB) [1] [2] that was first measured in 1998 [3]. The Kondo phenomenon is well known since the sixties and explains an anomaly in the temperature dependence of the resistivity of diluted magnetic alloys [4] [5].

This review is devoted to some features of equilibrium Kondo conductance across a quantum dot in the CB regime interacting with two contacts. It is remarkable here that the dot acts as a single impurity probed by the metal contacts, so that the properties of the Kondo state are not mediated over many impurities per cubic centimeter as it happens in diluted magnetic alloys. A strongly coupled state sets in between the dot and the contacts and phase coherence is established among the conduction electrons and the whole structure.

The temperature scale for the interactions between a magnetic impurity and the delocalized conduction electrons of the host metal is the so called Kondo temperature $T_{K}$. It is defined as the temperature at which the perturbative analysis breaks down. Therefore, different approaches are required to investigate the thermodynamics and the transport properties of a quantum dot in the Kondo regime in the whole range of temperatures from the perturbative region down to the unitarity limit.

Recently, the Kondo model and the Anderson impurity model in its Kondo limit have been deeply investigated by numerical renormalization group (NRG) calculations [6], the Bethe ansatz method [7] and conformal field theory (CFT) techniques [8]. Further developments in the NRG methods have been applied successfully in the crossover region $T \approx T_{K}$ [9]. The zero field spectral and transport properties of the Anderson model in the Kondo regime [10] as well as the field and temperature dependence of the Kondo resonance and the equilibrium magnetoconductance of the dot [11] have been investigated. The tunneling conductance as a function of the gate voltage has also been calculated with NRG, in wide temperature range for a single quantum dot with Coulomb interactions, assuming that two orbitals were active for the tunneling process [12]. 
We leave out the case when the electron distribution is not in local equilibrium about the Kondo impurity and the linear response theory is no longer sufficient. A number of techniques have been applied to describe nonequilibrium properties such as the nonlinear conductance, the nonequilibrium stationary state or the full time development of an initially out-of-equilibrium system: variational calculations [13], perturbation theory [14], equation of motion [15], perturbative functional integral methods [16], non-crossing approximation (NCA) [17] [18], perturbative renormalization group (RG) method in real time [19]. In particular, the last technique is well suited to describe quantum fluctuations which are induced by strong coupling between a small quantum system and an environment. It succeeds in reproducing the anomalous line shapes of the conductance observed in several recent experiments [3], due to the renormalization of the resistance and the local energy excitations [20].

We also leave out situations in which the levels localized at the dot are close in energy to the Fermi energy of the contacts (mixed valence models).

The plan of the paper is the following. We start from the tunneling hamiltonian formalism when the coupling between the dot and the contacts is weak (Section $I I$ ). A scattering approach in one-dimension is particularly suitable when studying the linear conductance in the device. The prototype model to account for strong electronelectron repulsion on the dot is the Anderson Hamiltonian with onsite repulsion (Section $I I I$ ). In the limit of strong correlation between the dot and the leads the latter model maps onto the so called "Kondo Hamiltonian model" (Section $I V$ ). A poor man's scaling approach up to second order leads us to the definition of the Kondo temperature $T_{K}$. Next, we introduce the physics of the single channel (Section $V I$ ) and the two channel Kondo problem (Section $V I I)$, both in the perturbative region and in the unitarity limit. In such a context we briefly sketch the Anderson, Yuval and Hamann Coulomb Gas approach [21] to the isotropic and anisotropic one channel Kondo system which gives a straightforward, though qualitative, insight of the strongly correlated state.

In the conventional setting the dynamical variable which is coupled to the electrons propagating from the leads is the total dot spin. There are cases in which the spin is locked to orbital degrees of freedom or even absent ("orbital Kondo") [22]; such cases are better found in a vertical geometry in presence of a magnetic field orthogonal to the dot [23]. A rather strong vertical magnetic field can induce level crossings in the dot due to orbital effects and produce accidental degeneracies which can give rise to exotic Kondo couplings (see Section VI.B $-C$ ). Some attention is drawn to the cylindrical geometry and to symmetry selection rules in the cotunneling process, also in connection with proposals for achieving the two channel Kondo non Fermi liquid fixed point in a vertical dot device [24] [25] [26] (Section VII.C).

\section{TUNNELING HAMILTONIAN AT WEAK COUPLING}

Quantum dots (QD) are fabricated in semiconductor heterostructures, by applying metallic gates to confine few electrons [27]. Because the confining area is quite small (the radius is $\sim 100 \div 1000 \AA$ ), the charging energy is much larger than the energy associated with thermal fluctuations, provided temperature is below $1^{\circ} \mathrm{K}$. Therefore the dot is only weakly linked to metal contacts and one can bias the system in such a way that the electron number $N$ in the dot can be changed at will. Because of the confining potential, dots display a level structure organized in shells, exactly the same as atoms do. Analyzing this level structure is of primary interest because these few interacting electrons $(N \leq 20)$ confined in a disk-like box (see Fig. 1), at special values of the parameters, are ruled by strong many-body Coulomb correlations. Hund's rules can be seen at work: the total spin $S$ of the electrons confined in the dot is maximized as long as no magnetic field is applied. On the other hand, a magnetic field in the direction orthogonal to the disk produces strong orbital effects which favour larger values of the total angular momentum $M$ as well as total spin $S$.

Quantum dots are remarkable because of Coulomb oscillations. At very low temperature linear conductance (at vanishing bias $V_{s d}$ ) is zero, except for peaks at discrete values of the gate voltage $V_{g}$, when it is energetically favourable to add one extra electron to the dot. Therefore $V_{g}$ controls the number of particles on the dot in the CB regime.

We tune the chemical potential $\mu$ of the left $(\mathrm{L})$ and right $(\mathrm{R})$ bulk contacts within the $\mathrm{CB}$ valley of the conductance at $N$ particles, that is $\mu_{N}<\mu<\mu_{N+1, \alpha} \equiv{ }^{N+1} E_{\alpha}-{ }^{N} E_{0}$; here the energies ${ }^{N \pm 1} E_{\alpha}$ are the total energies for the dot with $N \pm 1$ particles characterized by the quantum numbers $\alpha$ and ${ }^{N} E_{0}$ is the ground state energy (GS) at $N$ particles. If $N$ is odd, then the GS is at least doubly degenerate because the odd particle state can have spin $\sigma=\uparrow, \downarrow$. In this case the dot can be treated as a single Anderson impurity.

The peculiarity of these artificial atoms is in that the level structure can be investigated by measuring the tunneling current. Peaks in the linear conductance versus gate voltage $V_{g}$ separate regions at Coulomb blockade $(\mathrm{CB})$ in which $N$ is fixed and differs by just one electron. The peaks at zero source-drain voltage occur whenever $V_{g}$ compensates the required chemical potential for electron addition or subtraction. Hence, measuring a tunnel current across the 
device vs $V_{g}$ provides the spectroscopy of the dot levels. Adding a magnetic field the spin state of the dot can be changed, which in turn changes selection rules for electron tunneling [28].

The height and the width of the conductance peaks at resonance depends on temperature $T$. Let the dimensionless conductance be $g$ (in units of $2 \frac{e^{2}}{h}$ (factor of 2 is for the spin)). In the simplest case the maximum of the conductance at the resonant peak is $g_{\max } \propto \Gamma /\left(k_{B} T\right)$ and the halfwidth of the peak $\Gamma=\pi \nu(0)|V|^{2}$ is $\propto T$; here $V$ is the tunneling strength (see below) and $\nu(0)$ is the density of states at the Fermi energy. In the CB region tunneling via virtual states of the dot with $N \pm 1$ is only fourth order perturbation in $V$ (they are named cotunneling processes), $g \propto e^{2} \pi\left(\frac{2 \nu(0)|V|^{2}}{\epsilon_{d}}\right)^{2} / \hbar$, and vanishes exponentially with reducing temperature.

Nevertheless, unexpectedly, transport measurement in a dot gives rise to some new physics which can be related to the Kondo effect of spin impurities in non magnetic metal alloys (see Fig. 2) [3].

In the following we introduce the model for a quantum dot interacting with the contacts and define an equivalent one-dimensional Hamiltonian.

\section{A. Current within the tunneling Hamiltonian}

In this Subsection we discuss the mutual influence between the contacts and the dot, starting from the weak link limit. Conduction electrons in the leads constitute a Fermi sea (FS) of non interacting fermions with plane wave single particle wavefunctions on side $L$ and on side $R$. The dot is described by an Hamiltonian $H_{D}$ and coupling between the dot and the leads is accounted for through a tunneling term, so that the Hamiltonian describing the whole system is:

$$
H=H_{D}+\sum_{k \sigma} \epsilon_{k \sigma}^{R} a_{k \sigma}^{\dagger} a_{k \sigma}+\sum_{k \sigma} \epsilon_{k \sigma}^{L} b_{k \sigma}^{\dagger} b_{k \sigma}+\sum_{k \alpha \sigma}\left[V_{k \alpha} c_{k \alpha \sigma}^{\dagger} d_{\sigma}+V_{k \alpha}^{*} d_{\sigma}^{\dagger} c_{k \alpha \sigma}\right]
$$

The right and left Fermi sea (FS) of the two contacts $R, L$ are acted on by operators $c_{k R \sigma} \equiv a_{k \sigma}$ and $c_{k L \sigma} \equiv b_{k \sigma}$ ( here the index $\alpha$ stands for $R, L)$. The canonical transformation of the dot problem made by Glazman and Raikh [1] is just the construction of two species of fermions whose wavefunction is even or odd with respect to the dot center, in the case that the two barriers are unequal $V_{\alpha}=V_{R}, V_{L}$. It changes the picture from operators $a_{k \sigma}$ and $b_{k \sigma}$ to operators $\alpha_{k \sigma}$ and $\beta_{k \sigma}$ given by

$$
\begin{aligned}
\alpha_{k \sigma} & =u a_{k \sigma}+v b_{k \sigma}, \quad \beta_{k \sigma}=u b_{k \sigma}-v a_{k \sigma} \\
u & =\frac{V_{R}}{V}, \quad v=\frac{V_{L}}{V}, \quad V=\sqrt{\left|V_{R}\right|^{2}+\left|V_{L}\right|^{2}}, \quad \Gamma_{R, L}=\pi\left|V_{R, L}\right|^{2} \nu(0)
\end{aligned}
$$

where $\nu(0)$ is the density of states at the Fermi energy.

A general formula for the the conductance of interacting systems [30] [15] [31] has been written, resorting to the nonequilibrium Keldysh formalism [32]; in such a framework the current through the interacting region is written in terms of the distribution functions in the leads and local properties of the intermediate region, such as the occupation and the density of states at the dot site:

$$
J=\frac{i e}{2 h} \int d \omega\left(\operatorname{Tr}\left\{2\left[f_{L}(\omega) \Gamma_{L}-f_{R}(\omega) \Gamma_{R}\right]\left(\mathbf{G}^{r}-\mathbf{G}^{a}\right)\right\}+\operatorname{Tr}\left\{2\left(\Gamma_{L}-\Gamma_{R}\right) \mathbf{G}^{<}\right\}\right),
$$

where $\mathbf{G}^{r}, \mathbf{G}^{a}, \mathbf{G}^{<}$are the usual retarded, advanced, Keldysh Green functions for the dot in interaction with the leads and the $f$ are the Fermi functions. The Green's function $\mathbf{G}^{r}$ will be denoted as $G_{d d}$ in the following. Both $\mathbf{G}^{r}$ and $\Gamma_{R, L}$ are matrices in case that many channels are present.

Formula (3) can be cast in a more simple form in the case that the couplings to the leads differ only by a constant factor [15]:

$$
J=-2 \frac{e}{h} \int d \omega\left[f_{L}(\omega)-f_{R}(\omega)\right] \Im m \operatorname{Tr}\left\{\tilde{\Gamma} \mathbf{G}^{r}(\omega)\right\}
$$

where $\tilde{\Gamma}=\Gamma_{R} \Gamma_{L} / \Gamma$. Here $\Gamma=\Gamma_{R}+\Gamma_{L}$.

Now we derive explicitely $G_{d d}$ within the lowest order perturbation in the tunneling.

We start from the Hamiltonian in eq. (1), where $H_{D}$ is given by a single impurity energy level $\epsilon_{d}$. All quantities will be scalar quantities for simplicity. We define the electron retarded Green's function for the unperturbed leads: $G_{0}^{-1}=\sum_{k}\left(\omega-\epsilon_{k}+i 0^{+}\right)$. Projecting the equations for the total Green's function: 


$$
\begin{aligned}
\left(i \omega_{n}-H\right) G\left(i \omega_{n}\right) & =\mathbf{1} \\
G^{\dagger}\left(i \omega_{n}\right)\left(-i \omega_{n}-H\right) & =\mathbf{1}
\end{aligned}
$$

onto states in which one single extra particle is occupying the delocalized state $\mid k>$ or the impurity state $\mid d>$ we have:

$$
\begin{aligned}
\left(i \omega_{n}-\epsilon_{k}\right) G_{k, k^{\prime} \sigma}\left(i \omega_{n}\right) & =\delta_{k k^{\prime}}+V_{k} G_{d, k^{\prime} \sigma}\left(i \omega_{n}\right) \\
G_{d, k^{\prime} \sigma}\left(i \omega_{n}\right)\left(-i \omega_{n}-\epsilon_{k^{\prime}}\right) & =G_{d, d \sigma}\left(i \omega_{n}\right) V_{k^{\prime}}^{*}
\end{aligned}
$$

where $G_{d, k \sigma}\left(i \omega_{n}\right)=\left\langle d|G| k>, G_{k, k \sigma}\left(i \omega_{n}\right)=<k|G| k>, G_{d, d \sigma}\left(i \omega_{n}\right)=<d|G| d>\right.$ and $V_{k}=\langle k|V| d\rangle$.

This gives for each scattering channel (here in order to simplify the notation we suppress the channel label $\alpha$ ):

$$
\begin{aligned}
G_{k, k^{\prime} \sigma}\left(i \omega_{n}\right) & =\frac{\delta_{k k^{\prime}}}{i \omega_{n}-\epsilon_{k}}+\frac{V_{k}}{i \omega_{n}-\epsilon_{k}} G_{d, d \sigma}\left(i \omega_{n}\right) \frac{V_{k^{\prime}}^{*}}{i \omega_{n}-\epsilon_{k^{\prime}}} \\
& \equiv G_{k \sigma}^{0} \delta_{k k^{\prime}}+G_{k \sigma}^{0} V_{k} G_{d, d \sigma} V_{k^{\prime}}^{*} G_{k^{\prime} \sigma^{\prime}}^{0} .
\end{aligned}
$$

The density of states of the scattering electrons is defined as:

$$
\nu(\omega)=-\frac{1}{\pi} \Im m \sum_{k} \mathbf{G}_{k, k \sigma}^{r}(\omega) .
$$

Using similar equations for the dot, we write:

$$
G_{d, d \sigma}\left(i \omega_{n}\right)=\frac{1}{i \omega_{n}-\epsilon_{d}-\sum_{k}\left|V_{k}\right|^{2} /\left(i \omega_{n}-\epsilon_{k}\right)} .
$$

Now, let us consider the $L$ and $R$ contacts as two equal Fermi gases of noninteracting particles and equal chemical potential $\mu=0$ (within linear conductance regime a source drain voltage $V_{s d}$ is not applied). In such a case it is enough to discuss a single effective contact and the corresponding wavefunctions are plane waves of wavevector $k$ in the $x$-direction with a label $\alpha$ which includes all other quantum numbers. Their energy dispersion $\epsilon_{k \alpha}$ can be linearized about $\mu$ with $\epsilon_{q} \approx \hbar v_{F} q$, where $q$ is the momentum measured with respect to the Fermi momentum and $v_{F}$ is the Fermi velocity. Using a constant density of states $\nu(0)$ (at the Fermi energy per spin $L_{o} / 2 \pi \hbar v_{F}$ where $L_{o}$ is the size of the system) and a bandwidth of size $2 D$ and neglecting the $k$ dependence of $V_{k}$, the sum in eq. (9) is readily done:

$$
\sum_{k} \frac{\left|V_{k}\right|^{2}}{i \omega_{n}-\epsilon_{k}}=|V|^{2} \nu(0) \int_{-D}^{D} d \epsilon \frac{1}{i \omega_{n}-\epsilon}=\frac{\Gamma}{\pi} \ln \frac{i \omega_{n}+D}{i \omega_{n}-D} \equiv \Sigma_{d}\left(i \omega_{n}\right)
$$

The retarded Green's function for the dot is obtained from $G_{d, d \sigma}\left(i \omega_{n}\right)$ in the limit to real frequencies: $i \omega_{n} \rightarrow \omega+i 0^{+}$ according to:

$$
\mathbf{G}_{d, d \sigma}^{-1}\left(i \omega_{n}\right)=\left\{i \omega_{n}-\epsilon_{d}-\Sigma_{d}\left(i \omega_{n}\right)\right\} \rightarrow \omega-\epsilon_{d}-\frac{\Gamma}{\pi} \ln \left|\frac{v_{F} D+\omega}{v_{F} D-\omega}\right|+i \Gamma=\omega-\widetilde{\epsilon}_{d}+i \Gamma,
$$

where $\tilde{\epsilon}_{d}$ is the renormalized dot energy. The coupling of the dot to the contacts shifts the location of the pole corresponding to the energy of the localized level and gives a finite width $\Gamma$ to the resonance.

\section{B. 1-d Scattering formalism}

At zero temperature scattering is only elastic. In the following we develop a $1-d$ scattering approach which is mostly useful in vertical structures [33], next we show that also the tunneling conduction can be cast into the scattering formalism.

If the evolution operator $U\left(t, t^{\prime}\right)$ is known, the transmission amplitude can be written in a scattering approach as $\theta^{R \rightarrow L}=<b^{\dagger} U(+\infty,-\infty) a>=v^{*} u<\alpha^{\dagger}(\infty) \alpha(-\infty)>-u^{*} v<\beta^{\dagger}(\infty) \beta(-\infty)>\equiv v^{*} u S^{0}+u^{*} v S^{1}$, where we have defined the two expectation values as the scattering matrix elements for the two uncoupled even and odd channels. Hence, the conductance takes the form of the Landauer formula: 


$$
g=T^{R \rightarrow L} \equiv\left|\theta^{R \rightarrow L}\right|^{2}=4\left|v^{*} u\right|^{2}\left|\frac{1}{2} \sum_{l} S^{l}\right|^{2}=\frac{4 \Gamma_{R} \Gamma_{L}}{\left(\Gamma_{R}+\Gamma_{L}\right)^{2}}\left|\frac{1}{2} \sum_{l} S^{l}\right|^{2} .
$$

The potential after the transformation of eq. (2) has become even, hence unitarity is satisfied by each channel individually: $\left|S^{l}\right|^{2}=1, l=0,1$. The unitary limit of the conductance is $g_{u}=\frac{4 \Gamma_{R} \Gamma_{L}}{\left(\Gamma_{R}+\Gamma_{L}\right)^{2}}$. In particular, if the potential is $\delta(x)$, odd parity is totally transmitted and such that $S^{1}=-1$.

Eq. (12) is valid for a general system with an interacting intermediate region. One can match this approach to a 1-d scattering approach for non interacting electrons as it is done here below.

Let us place the impurity (QD) at $x=0$ and consider the scattering amplitude $f_{L, R}$ of a one electron wavefunction $\psi(x)$

$$
\begin{array}{ll}
\psi_{>}(x) \propto e^{i k x}+f_{R} e^{i k x} & x>>0 \\
\psi_{<}(x) \propto e^{i k x}+f_{L} e^{-i k x} & x<<0 .
\end{array}
$$

Here the transmission coefficient is $T=\left|1+f_{R}\right|^{2}$ while the reflection coefficient is $R=\left|f_{L}\right|^{2}$ and they satisfy the conservation of flux: $T+R=1$.

If the dot structure is even with respect to the origin along the vertical axis, the even parity $l=0$ and the odd parity $l=1$ channels are independent. It is then useful to define even and odd parities $f^{l}: f^{0}=\frac{1}{2}\left(f_{L}+f_{R}\right), f^{1}=\frac{1}{2}\left(f_{R}-f_{L}\right)$ and the elastic $t$ - matrix $t^{l}=i k_{o} f^{l} / \pi$. Here the energy of the incoming particle is $\hbar v_{F} k_{o}$ (in units $\hbar v_{F}=1$, it follows that $[t]=$ energy) and $k_{o}=2 \pi / L_{o}$ (where $L_{o}$ is the linear size of the system). The $t$ - matrix is related to the $S-$ matrix according to:

$$
\begin{aligned}
S^{l} & =e^{2 i \delta^{l}} ; \quad S^{l}-1=-\frac{2 \pi i}{k_{o}} t^{l} \\
t^{l} & =-\frac{k_{o}}{\pi} \sin \delta^{l} e^{i \delta^{l}}
\end{aligned}
$$

where $\delta^{l}$ are the phase shifts for the two parities. In this context unitarity of the $S$-matrix, $\frac{1}{2} \sum_{l}\left|S^{l}\right|^{2}=1$, is the same as flux conservation $R+T=1$.

Conductance is given by the Landauer formula:

$$
\text { conductance }=g=T=\left|1+f_{R}\right|^{2}=\left|1+i \sum_{l} \sin \delta^{l} e^{i \delta^{l}}\right|^{2} .
$$

In the case of resonant tunneling we have $T \sim 1$ and $R=\left|f_{L}\right|^{2} \sim 0$, so that eq. (15) becomes $g \rightarrow 1$, that is the unitary limit.

The condition $R=\left|\pi\left(t^{0}-t^{1}\right) /\left(i k_{o}\right)\right|^{2}=\sin ^{2}\left(\delta^{0}-\delta^{1}\right) \sim 0$ yields $\delta^{0} \sim \delta^{1} \equiv \delta \bmod \pi$, while in eq. (15) we have $T \sim 1$ for $\delta^{0}=\delta^{1} \equiv \delta \rightarrow \pi / 2$.

If the potential is even $\left(\Gamma_{R}=\Gamma_{L}\right)$, unitarity is satisfied by each channel individually: $\left|S^{l}\right|^{2}=1, l=0,1$; in particular, if the potential is $\delta(x)$, odd parity is totally transmitted and such that $S^{1}=-1$, and the conductance becomes:

$$
g=\left|\frac{1}{2}\left(S^{0}-1\right)\right|^{2}=\left|\frac{\pi}{k_{o}} t^{0}\right|^{2}=\sin ^{2} \delta^{0} .
$$

Again, if $\delta^{0}=\pi / 2$ conductance reaches the unitarity limit.

In the following we describe the basic approximations and the weak coupling limit of very opaque barriers between dot and contacts.

In this case tunneling of lead electrons across the dot is a perturbative process as derived in Section IIA. Within such a perturbative regime neither the dot nor the contacts are much affected by the interaction with the other. The relevant effect on the QD is the shifting of its levels and a level broadening, which is a second-order effect in the tunneling strength $V$. This shows itself as a small imaginary part added to the energy levels, i.e. a finite lifetime.

It is easy to show that the linear conductance given above can be rephrased in terms of the imaginary part of the Green's function as in eq. (4) at the perturbative level. To this end, let us first state some formalities regarding the selfenergy $\boldsymbol{\Sigma}$ and the $\mathbf{t}$ matrix:

$$
\left(E-H_{0}-\Sigma\right) \mathbf{G}=\mathbf{1} ; \mathbf{G}_{0}\left(E-H_{0}\right)=\mathbf{1} \rightarrow \mathbf{G}_{0} \mathbf{G}^{-1}+\mathbf{G}_{0} \mathbf{\Sigma}=\mathbf{1} \rightarrow \mathbf{G}=\mathbf{G}_{0}+\mathbf{G}_{0} \mathbf{\Sigma} \mathbf{G} .
$$


In the Born approximation $\Sigma$ and $\mathbf{t}$ coincide because by definition is $\mathbf{G}=\mathbf{G}_{0}+\mathbf{G}_{0} \mathbf{t} \mathbf{G}_{0}$. We assume that in tunneling the odd parity channel is fully transmitted $\left(S^{1}=-1\right)$ and the even parity one gives (see eq. (7)):

$$
\begin{aligned}
t^{0} & \sim V G_{d, d} V, \quad G_{d, d}=\frac{1}{\epsilon-\widetilde{\epsilon}_{0}+i \Gamma} \\
& \rightarrow t^{0} \sim \frac{|V|^{2}}{\epsilon-\widetilde{\epsilon}_{0}+i \Gamma}, \quad \frac{\Im m t^{0}}{\Re e t^{0}}=\tan \delta^{0}=\frac{-\Gamma}{\epsilon-\widetilde{\epsilon}_{0}}, \\
\sin ^{2} \delta^{0} & =\frac{\tan ^{2} \delta^{0}}{1+\tan ^{2} \delta^{0}}=\frac{\Gamma^{2}}{\left(\epsilon-\widetilde{\epsilon}_{0}\right)^{2}+\Gamma^{2}} .
\end{aligned}
$$

Here $V$ is the tunneling matrix element defined in eq. (2), $\widetilde{\epsilon}_{0}$ is the renormalized quantity defined in eq. (11) and $\Gamma=\Gamma_{R}+\Gamma_{L}$ is the inverse lifetime of the resonance. This implies that eqs. $(12,16)$ become:

$$
g=g_{u} \sin ^{2} \delta^{0}=\frac{4 \Gamma_{R} \Gamma_{L}}{\left(\epsilon-\widetilde{\epsilon}_{0}\right)^{2}+\Gamma^{2}},
$$

where $g_{u}=\frac{4 \Gamma_{R} \Gamma_{L}}{\left(\Gamma_{R}+\Gamma_{L}\right)^{2}}$. On the other hand, because $\Im m G=-\Gamma /\left[\left(\epsilon-\widetilde{\epsilon}_{0}\right)^{2}+\Gamma^{2}\right]$, eq. (4) becomes:

$$
g=\int d \omega\left(-\frac{\partial f(\omega)}{\partial \omega}\right) \tilde{\Gamma} \frac{\Gamma}{\left(\epsilon-\widetilde{\epsilon}_{0}\right)^{2}+\Gamma^{2}}
$$

where $\tilde{\Gamma}=\Gamma_{R} \Gamma_{L} / \Gamma$. At zero temperature both results of eqs. (18) and (19) coincide.

At finite temperature, if the odd channel is fully transmitted eq.(12) can be generalized as

$$
g=g_{u} \int d \omega\left(-\frac{\partial f(\omega)}{\partial \omega}\right)\left[-\pi \nu(0) \Im m\left(t^{0}\right)\right]
$$

where $t^{0}$ is the $\mathbf{t}$ matrix above defined and related to the exact retarded Green function through the relation $\mathbf{G}=$ $\mathbf{G}_{0}+\mathbf{G}_{0} \mathbf{t} \mathbf{G}_{0}$. We have used the optical theorem:

$$
\frac{\pi^{2}}{k_{o}^{2}} \operatorname{Tr}\left\{t^{0} t^{0 \dagger}\right\}=\Re e\left\{\frac{i \pi}{k_{o}} t^{0}\right\}
$$

which follows from the unitarity condition $\left|S^{0}\right|^{2}=1$.

\section{ROLE OF THE ONSITE REPULSION $U$ IN TUNNELING}

Up to now $H_{D}$ referred just to a single impurity level $\epsilon_{d}$. Indeed, charging energy $U$ is the main feature of a $Q D$ and we have to deal with it. Something which is closer to a $Q D$ is an impurity level with onsite repulsion $U$. Inclusion of $U$ in the Hamiltonian (1) leads to the single level Anderson model:

$$
H_{\mathrm{AND}}=H_{\text {lead }}+\epsilon_{d} \sum_{\sigma} n_{\sigma}+U \sum_{\sigma \sigma^{\prime}} n_{\sigma} n_{\sigma^{\prime}}+\sum_{k \alpha \sigma} V_{\alpha}\left[c_{k \alpha \sigma}^{\dagger} d_{\sigma}+d_{\sigma}^{\dagger} c_{k \alpha \sigma}\right] .
$$

If $\epsilon_{d}=-U / 2$ with respect to the chemical potential of the conduction electrons $\mu$ (taken as the zero of the single particle energies), the Anderson model which arises is symmetric. In fact, the energies of the empty impurity state, ${ }^{0} E$, and of the doubly occupied impurity state, ${ }^{2} E=2 \epsilon_{d}+U$, are both zero, while the singly occupied impurity level has energy ${ }^{1} E=-U / 2$.

In order to understand how the Coulomb repulsion on the dot site affects the Green's function of the dot we use a path integral formalism. We show that, in the limit in which the charge degree of freedom on the dot is frozen $(U \rightarrow \infty)$, the dot Green's function describes the dynamics of the only degree of freedom left, that is the dot magnetization $\left\langle n_{\uparrow}-1 / 2>\equiv<1 / 2-n_{\downarrow}>\right.$ which is forced by a stochastic field $X(\tau)$ in imaginary time, produced by the scattering of the lead electrons, with a gaussian probability distribution. The excitation energy associated to it is shifted from $\epsilon_{d}$ to the Fermi level: this is the origin of the resonance at the Fermi level in the Kondo problem.

As a first step, we will rephrase the previous result of an impurity with $U=0$ in this new approach. After linearizing the bands, the leads action can be written in terms of the field operators $\psi_{u \alpha}$ (where $\alpha=L, R$ and $u$ includes all other quantum numbers) as: 


$$
S_{\text {lead }}=-i v_{F} \int_{0}^{\beta} d \tau \int d x \sum_{u} \sum_{\alpha=L, R} \psi_{u \alpha}^{\dagger}(x, \tau) \frac{d}{d x} \psi_{u \alpha}(x, \tau) .
$$

With respect to the tunneling action, if the $L$ and $R$ barriers are equal and the dot is zero dimensional the interaction term only includes the symmetric combinations $\Phi_{u}(\tau)=\frac{1}{\sqrt{2}}\left(\psi_{u L}(x=0, \tau)+\psi_{u R}(x=0, \tau)\right)$ at the origin:

$$
S_{\mathrm{T}}=\int_{0}^{\beta} d \tau \sqrt{2} \sum_{u}\left[V \Phi_{u}^{\dagger}(\tau) d_{u}(\tau)+V^{*} d_{u}^{\dagger}(\tau) \Phi_{u}(\tau)\right]
$$

The total action is:

$$
\mathcal{A}=\int_{0}^{\beta} d \tau\left\{\sum_{u} d_{u}^{\dagger}(\tau)\left[\frac{\partial}{\partial \tau}+\left(\epsilon_{d}-\mu\right)\right] d_{u}(\tau)\right\}+S_{\text {lead }}+S_{\mathrm{T}}
$$

One can first integrate out the degrees of freedom of the $\psi_{u \alpha}(\tau, x)$ fields for $x \neq 0$ which are free-like, and next the field $\Phi_{u}$ at $x=0$ which is the only one interacting with the dot variable $d_{u}(\tau)$. Because the fields in the leads are non interacting, the result of the gaussian integration yields an effective action for the dot:

$$
-S_{\mathrm{D}}^{\mathrm{Eff}} \propto \ln \left\{\int \prod_{\alpha=L, R} \prod_{u} \mathcal{D} \psi_{u \alpha} \mathcal{D} \psi_{u \alpha}^{\dagger} e^{-\mathcal{A}}\right\}=-\beta \sum_{i \omega_{n}} d_{u}^{\dagger}\left(i \omega_{n}\right)\left(i \omega_{n}-\epsilon_{d}-\Sigma\left(i \omega_{n}\right)\right) d_{u}\left(i \omega_{n}\right)
$$

where the self-energy correction to the Green's function of the dot $\Sigma\left(i \omega_{n}\right)=\frac{\Gamma}{\pi} \ln \left(\frac{i \omega_{n}+D}{i \omega_{n}-D}\right)$ was obtained in eq. (10).

\section{A. Coulomb repulsion on the dot: freezing of the charge degree of freedom}

We now discuss the role of the onsite Coulomb interaction. In the large- $U$ limit we have:

$$
\begin{aligned}
\exp \int_{0}^{\beta} d \tau\left\{-\epsilon_{d}\left(n_{\uparrow}+n_{\downarrow}\right)-U n_{\uparrow} n_{\downarrow}\right\} & =e^{-\frac{U}{4} \int_{0}^{\beta} d \tau\left\{\left(n_{\uparrow}+n_{\downarrow}\right)^{2}+\frac{4}{U} \epsilon_{d}\left(n_{\uparrow}+n_{\downarrow}\right)\right\}} \cdot e^{\frac{U}{4} \int d \tau\left(n_{\uparrow}-n_{\downarrow}\right)^{2}} \\
& =e^{\frac{\beta}{U} \epsilon_{d}^{2}} \delta\left(n_{\uparrow}+n_{\downarrow}+2 \epsilon_{d} / U\right) \cdot e^{\frac{U}{4} \int_{0}^{\beta} d \tau\left(n_{\uparrow}-n_{\downarrow}\right)^{2}},
\end{aligned}
$$

where the delta function, which is defined by the last equality in the limit $U \rightarrow \infty$, implements the constraint of single site occupancy in the symmetric case, $\epsilon_{d}=-U / 2$.

The quartic interaction is decoupled by means of a Hubbard-Stratonovitch boson field $X(\tau)$, according to the identity:

$$
e^{\frac{U}{4} \int_{0}^{\beta} d \tau\left(n_{\uparrow}-n_{\downarrow}\right)^{2}}=\int D X e^{-\frac{1}{4 U} \int_{0}^{\beta} d \tau\left(X^{2}(\tau)+2 U\left(n_{\uparrow}-n_{\downarrow}\right) X(\tau)\right)} .
$$

Having introduced the field $X(\tau)$, the partition function $\mathcal{Z}(\mu)$ takes the form:

$$
\begin{aligned}
\mathcal{Z}(\mu) \propto & \int D X e^{-\frac{1}{4 U} \int_{0}^{\beta} d \tau X^{2}(\tau)} \\
& \times \int \Pi_{i}\left(D d_{i} D d_{i}^{\dagger} e^{-\int_{0}^{\beta} d \tau d \tau^{\prime} d_{i}^{\dagger}(\tau) G_{(0)}^{-1}\left(\tau-\tau^{\prime}\right) d_{i}\left(\tau^{\prime}\right)}\right) \\
& \times \delta\left(n_{\uparrow}+n_{\downarrow}-1\right) \cdot \frac{1}{2} \sum_{j=\uparrow, \downarrow} e^{(-1)^{j} \int_{0}^{\beta} d \tau\left[n_{j}-\frac{1}{2}\right] X(\tau)}
\end{aligned}
$$

Note that now the term $\epsilon_{d} \sum_{i} n_{i}$ was included in eq. (27), so in this case the dot Green's function is $G_{(0)}^{-1}\left(i \omega_{n}\right)=$ $i \omega_{n}-\Sigma_{(0)}\left(i \omega_{n}\right)$. At odds with the case $U=0$ here the resonance is at the Fermi level, in spite of the fact that the original localized level is at $\epsilon_{d}$. The partition function in eq. (28) describes an effective spin-1/2 coupled to the fluctuating magnetic field $X(\tau)$; its dynamics is constrained by the requirement that the impurity is singly occupied. It could be shown that the single site occupancy constraint is fulfilled in the average when the partition function of eq. (28) is used. 


\section{B. Quenching of the magnetic moment: singlet ground state}

The representation of the partition function given in eq. (28) allows us to recognize the doubly degenerate ground state (GS) of the impurity spin $S_{d}=1 / 2$ with $S_{d}^{z}=(-1)^{\sigma}\left(n_{\sigma}-1 / 2\right), \sigma=\uparrow, \downarrow$, driven by the field $X(\tau)$ and produced by virtual tunneling of electrons on and off the dot at energy $\mu$.

Anderson, Yuval and Hamann [21] calculated eq. (28) by integrating out the impurity ( $d, d^{\dagger}$ fields) and showing that the interaction with the delocalized electrons gives rise to a dynamics of the field $X(\tau)$ which also interacts with itself at different times according to a logarithmic law. The problem was solved by mapping eq. (28) onto the partition function of a Coulomb gas (CG) [34] of flips in $1-d$, as we show in some detail in this subsection.

Let us define the new field $\xi(\tau)=X(\tau) / \Gamma$, where $\Gamma=\pi \nu(0)|V|^{2}$ is related to the tunneling strength. At low temperatures, saddle point solutions of the resulting single particle effective action are sequences of instantons $\xi_{ \pm}(\tau, l)= \pm \xi_{M} \tanh \left(\left(\tau-\tau_{l}\right) / \tau_{M}\right)$ (where $\tau_{l}, l=1,2, \ldots$ are the centers and $\tau_{M} \sim \frac{1}{\epsilon_{F}}$ is the width, that is some cut-off which regularizes the theory at short times) corresponding to jumps between the two minima of the effective potential $V_{\text {eff }}[\xi]=\left(\Gamma^{2} / U\right) \xi^{2}-2 \Gamma / \pi\left[\xi \tan ^{-1}(\xi)-\frac{1}{2} \ln \left(1+\xi^{2}\right)\right]$, located at $\xi_{M}= \pm U / 2 \Gamma$, and interacting via a logarithmic potential $\alpha^{2} \ln \left|\left(\tau_{i}-\tau_{j}\right) / \tau_{M}\right|$.

For such a CG we can define the bare strength $\alpha_{b}^{2}$ of the logarithmic interaction (the "charge") as $\alpha_{b}^{2}=2(1-8 \Gamma / U \pi)$ and the "fugacity" $Y$ as $Y \equiv \tau_{M} e^{-S_{\text {tot }}}$.

Thus, the full partition function may be approximated with the sum over the trajectories given by hopping paths and will be written as:

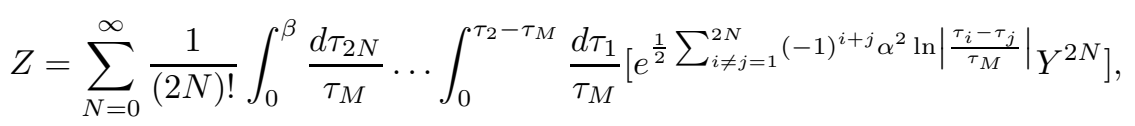

that is the partition function of an effective one-dimensional gas of spin flips. The integral over the "centers of the instantons" has to be understood such that $\tau_{i}$ and $\tau_{j}$ never become closer than $\tau_{M}$.

Now, we are ready to perform the RG analysis to get the behavior of the model at large time scales (low temperatures) [21]. The bare fugacity of the CG is $Y_{b}=\tau_{M} \exp (-\overline{\mathcal{A}})$ where $\overline{\mathcal{A}} \sim \tau_{M} U$ is the action of one single flip. The scaling of the fugacity and the renormalization of the coupling constant induced by processes of fusion of charges lead to the following Renormalization Group (RG) equations [34]:

$$
\frac{d Y}{d \ln \tau_{M}}=\left(1-\frac{\alpha^{2}}{2}\right) Y ; \frac{d \alpha^{2}}{d \ln \tau_{M}}=-2 Y^{2} \alpha^{2}
$$

(see Appendix A for a sketch on the derivation).

We see clearly that the flow is towards $Y \rightarrow \infty$ and $\alpha^{2} \rightarrow 0$ and the scaling invariant energy (that is the Kondo temperature which we will introduce in the next section) is $T_{K}=\tau_{M}^{-1} e^{-1 /\left(1-\frac{\alpha^{2}}{2}\right)} \sim(U \Gamma)^{\frac{1}{2}} e^{-\pi U /(8 \Gamma)}$.

Condensation of instantons in the doubly degenerate GS leads to the Kondo singlet $\left.<S^{z}\right\rangle=0$ on the dot. Now we present an heuristic argument for such quenching of the total spin $S$ on the dot; it runs as follows.

The dynamics of the field $\xi(\tau)$ with action $\overline{\mathcal{A}}$ can be mimicked by a two-level system hamiltonian $H_{2 l}$ with hopping energy $\lambda \sim \frac{1}{2} m_{\text {eff }}\left(\frac{d \xi}{d \tau}\right)^{2} \sim \frac{1}{2} \frac{\Gamma^{2}}{U} \tau_{M}^{2}\left(\xi_{M} / \tau_{M}\right)^{2} \sim U / 2$. The role of the interaction is to project out higher energy components from the dot states. Let us denote by $\mid \pm>$ the two eigenstates of $H_{2 l}$, i.e. the singlet and the triplet (with respect to the total spin of the dot plus conduction electrons, $S=0$ and $S=1$ ) on the dot. Instantons, by flipping the impurity spin, produce a dynamics of the system "dot + conduction electrons " between these two states.

Now, let us make an interesting analogy with thermodynamics. At temperature $\beta^{-1}$ the probabilities of having the system in one of the two states will be given by [35]:

$$
P_{+}=\frac{1}{1+e^{-2 \beta \lambda}} ; P_{-}=\frac{e^{-2 \beta \lambda}}{1+e^{-2 \beta \lambda}}
$$

partly eliminating the component on the high energy eigenstate $\mid->$. Thus we are ready to give the connection with the CG picture described by the partition function of eq. (29). In our case the dynamics is given by the coherent zero point fluctuations of the system as a whole. From the statistical weight associated to a configuration with $N$ instantons we can easily write down the formula:

$$
\langle N\rangle \equiv\left\langle N_{\text {inst }}\right\rangle=\frac{\sum_{N=0}^{\infty} 2 N Y^{2 N} /(2 N) !}{\sum_{N=0}^{\infty} Y^{2 N} /(2 N) !}=Y \frac{d}{d Y} \ln (\cosh Y)=Y \tanh Y .
$$


The frequency $\lambda / 2 \pi$ is obviously related to the average number of flips: $\beta \lambda / 2 \pi=<N\rangle=Y \tanh Y$, as a direct implication of eq. (31).

Hence, the probabilities of having the system in the states $\mid \pm>$ within the ground state $(T=0)$ are:

$$
P_{+}=\frac{1}{1+e^{-4 \pi Y \tanh Y}} ; P_{-}=\frac{e^{-4 \pi Y \tanh Y}}{1+e^{-4 \pi Y \tanh Y}} .
$$

Because $Y$ scales to infinity, the higher energy state completely decouples and the total spin on the dot is quenched: $<S^{z}>\rightarrow 0$.

\section{PERTURBATIVE ANALYSIS AT $T>>T_{K}$}

The Kondo Effect in metals containing magnetic impurities is responsible for the "anomalous" minimum in the resistivity $\rho(T)$, as the temperature $T \sim T_{K}$ : such a minimum in $\rho(T)$ is due to scattering of conduction electrons off the localized magnetic impurities. Those contributions were first worked out by Kondo [36], who derived a correction $\Delta \rho(T) \propto \ln \left(\frac{T_{K}}{T}\right)$.

As we pointed out in the introduction, a different realization of the Kondo effect may be achieved, in a controlled way, in a quantum dot in Coulomb Blockade (CB)-regime [3]. The Kondo effect in a dot is usually detected by measuring the linear conductance as a function of the gate voltage by connecting two electrodes to the dot. A dot at CB exhibits discrete energy levels. Changing the number of electrons is strongly prevented by electrostatic charging energy. Correspondingly, the total charge at the dot is quantized and the linear conductance is zero within large windows of variations of the gate voltage ("CB-valleys"). By analyzing the level structure of the dot it has been shown that, in some special cases, the dot at $\mathrm{CB}$ behaves as a magnetic moment antiferromagnetically interacting with the magnetic momenta of lead electrons. CB-valleys at different occupation number of the dot are bounded by resonant conduction peaks, as the chemical potential of the leads matches the one of the dot. Those peaks usually get sharper and better defined as $T$ is lowered. However, as $T \sim T_{K}$, Kondo effect arises at the dot. Consequently, the CB-valley between two resonant conduction peaks "fills in" in a way that does not depend on the position of the Fermi level of the leads [3]. As $T \sim T_{K}$, the conductance $g(T)$ exhibits the typical logarithmic raise [37]. The logarithmic raise, however, cannot hold all the way down to $T=0$, as it must be limited by the unitarity bound. Therefore, a different approach is required in order to study Kondo effect in the $T=0$-unitarity limit, as we will discuss in the next section.

In this Section we derive the Kondo Hamiltonian from the single impurity Anderson model (eq. 22) focusing for simplicity on the isotropic case $J_{\perp}=J_{z}=J$, then we sketch the perturbative Renormalization Group (RG) flow for the coupling strength of such a model. In general, the lower is $T$, the more spin-flip processes become likely, which make the running coupling constant $J$ grow. At the Kondo temperature $T_{K}$ the perturbative analysis breaks down, that is $\nu(0) J\left(T_{K}\right) \sim 1$, so that $T_{K}$ is the characteristic scale that divides the regions of weak and strong coupling.

\section{A. Derivation of the spin dynamics hamiltonian}

In the following we will restrict our analysis to a two-fold degenerate dot level: in such a case the QD can be modelized as a spin-1/2 magnetic impurity. Indeed, because the charge degree of freedom is frozen at Coulomb Blockade we mimick the dot with its total spin $\vec{S}_{d}$ and describe its interaction with the delocalized electrons by means of the Kondo Hamiltonian:

$$
H_{\text {eff }}=H_{\text {lead }}+H_{K} \equiv H_{\text {lead }}-J \vec{S}_{d} \cdot \vec{\sigma}(0),
$$

where $\vec{\sigma}(0)=\sum_{k k^{\prime}} \sum_{\sigma \sigma^{\prime}}\left(c_{k \sigma}^{\dagger} \vec{\tau}_{\sigma \sigma^{\prime}} c_{k^{\prime} \sigma^{\prime}}\right)$ plays the role of a spin density of the itinerant electrons at the impurity site $\left(\vec{\tau}_{\sigma \sigma^{\prime}}\right.$ are the Pauli spin- $\frac{1}{2}$ matrices). Its components behave as a spin- $\frac{1}{2}$, provided single occupancy of the site $x=0$ is guaranteed.

In the anisotropic Kondo model the couplings of the $x, y$ components $J_{\perp}$ are different from the one of the $z$ component $J_{z}$. This effective Hamiltonian is more suitable to describe the low- $T$ physics of the system because its dynamics shows how the system flows towards the nonperturbative regime. In the following sections by "low temperature" we'll mean $T \sim T_{K}$. 
As a first step we show that it is possible to derive the effective Hamiltonian (33) from the one for the single impurity Anderson model (eq. 22), where $H_{\mathrm{D}}=\epsilon_{d} \sum_{\sigma} n_{\sigma}+U \sum_{\sigma \sigma^{\prime}} n_{\sigma} n_{\sigma^{\prime}}$, by means of the Schrieffer-Wolff (SW) transformation [38]. More details can be found on the book by Hewson [5].

Let $\Xi$ be the space spanned by the (almost) degenerate dot's states. As the number of electrons at the QD is fixed, the relevant degrees of freedom can be described by an effective Hamiltonian $H_{\text {eff }}$ acting onto $\Xi$ only. In order to construct $H_{\text {eff }}$, we apply the SW transformation to the Hamiltonian in eq. (22). We denote by $P$ the projector onto $\Xi$ and by $\epsilon_{d}$ the energy of the states within $\Xi$, so that the effective Kondo Hamiltonian is given by:

$$
\delta H_{\mathrm{eff}} \approx P V(1-P) \frac{1}{\epsilon_{d}-H_{\mathrm{D}}-H_{\text {lead }}}(1-P) V P .
$$

Indeed, by inserting eq. (22) into eq. (34), we get the result:

$$
\begin{aligned}
H_{\mathrm{eff}}= & H_{\mathrm{lead}}-\nu(0) \sum_{\alpha \sigma} \frac{V_{\alpha}^{2}}{\epsilon_{d}} d_{\sigma}^{\dagger} d_{\sigma}+\sum_{\alpha \sigma ; k, k^{\prime}} V_{\alpha}^{2}\left[\frac{1}{\epsilon_{d}+U}+\frac{1}{\epsilon_{d}}\right] c_{k \alpha \sigma}^{\dagger} c_{k^{\prime} \alpha \sigma}+ \\
& -\sum_{\alpha, \alpha^{\prime} ; \sigma, \sigma^{\prime} ; k, k^{\prime}} V_{\alpha} V_{\alpha^{\prime}}\left[\frac{1}{\epsilon_{d}+U}-\frac{1}{\epsilon_{d}}\right] c_{k \alpha \sigma}^{\dagger} \vec{S}_{d} \cdot \vec{\tau}_{\sigma \sigma^{\prime}} c_{k^{\prime} \alpha^{\prime} \sigma^{\prime}}
\end{aligned}
$$

where $S_{d}^{z}=\sum_{\sigma} \sigma d_{\sigma}^{\dagger} d_{\sigma}, S_{d}^{+}=d_{\uparrow}^{\dagger} d_{\downarrow}$ and $S_{d}^{-}=d_{\downarrow}^{\dagger} d_{\uparrow}$ are the impurity spin components. Spin commutation relations are obtained if no double occupancy is admitted.

Besides $H_{\text {lead }}$, the first and the second term at the r.h.s. of eq. (35) represent, respectively, a renormalization of the dot's energies and a potential scattering term; the third term is the one which induces spin flips. The two contributions appearing in the potential scattering term and in the spin-flip term refer to two virtual particle and hole processes, respectively. In the first case a particle is added to the dot so that the energy $\epsilon_{d}+U$ is involved, while in the second case a particle is subtracted from the dot level thus paying the energy $\left|\epsilon_{d}\right|$. The potential scattering term vanishes if the Anderson model is symmetric, as we have assumed up to now $\left(\epsilon_{d}=-U / 2\right)$.

\section{B. Perturbative Renormalization Group Approach}

In this Subsection the starting point of our reasoning is, for simplicity, the isotropic limit of Kondo hamiltonian in eq. (33). Scattering of conduction electrons by the impurity produces a self energy correction, as well as a correction to the interaction vertex. The transitions between the states close to the Fermi level $\epsilon_{F}$ and the states within a narrow strip of energies of width $\delta D$ near the edges of the band of width $2 D$ are associated with an high energy deficit. Such transitions are virtual and their influence on the states near $\epsilon_{F}$ can be taken into account perturbatively in the second order. In the poor man's scaling approach one includes second order corrections arising from processes in which the electrons $k^{\prime}$ are scattered to an intermediate state at energy $D>\epsilon_{k^{\prime \prime}}>D-\delta D$ or $-D<\epsilon_{k^{\prime \prime}}<-D+\delta D$ where $D$ is some ultraviolet cut-off. Because they involve high intermediate energies, one can think of including them perturbatively, so for an electron $k^{\prime}$ scattered into the final state $k$ we have:

$$
\sum_{k^{\prime \prime} \in \gamma}<k\left|H_{K}\right| k^{\prime \prime}><k^{\prime \prime}\left|\frac{1}{E-H_{\text {lead }}}\right| k^{\prime \prime}><k^{\prime \prime}\left|H_{K}\right| k^{\prime}>\sim-J^{2} \nu(0) \delta D \frac{1}{D} \vec{S}_{d} \cdot \sum_{k k^{\prime}} \sum_{\sigma \sigma^{\prime}} c_{k \sigma}^{\dagger} \vec{\tau} \sigma \sigma^{\prime} c_{k^{\prime} \sigma^{\prime}}
$$

where $\gamma$ is the $k^{\prime \prime}$ domain mentioned above. To justify the second step one observes that, in the shell $\gamma$, the operator $H_{\text {lead }}$ can be replaced by an energy $D$ and, in comparison to it, the eigenvalue $E$ can be put at the Fermi level $E=0$. The result is an effective Hamiltonian acting within the band of a reduced width $D-\delta D$, of the same form as $H_{K}$ in eq. (33) but with a modified value of $J$. This gives the following correction to the previous coupling constant in $H_{K}$ : $\delta J \sim-J^{2} \nu(0) \delta D / D$ [39]. Successive reductions of the bandwidth by small steps $\delta D$ can be viewed as a continuous process during which the initial Hamiltonian is trasformed to an effective low-energy Hamiltonian acting within the band of reduced width $D-\delta D$. The evolution of the exchange amplitude $J$ during such a poor man's scaling can be cast into the form of a flow differential equation [39]:

$$
\frac{d J}{d \ln D}=-\nu(0) J^{2}
$$

Integration of eq. (37) gives rise to the renormalization of $J$ : 


$$
\frac{1}{j(D)}-\frac{1}{j\left(D_{0}\right)}=\ln \frac{D}{D_{0}}
$$

where $j=J \nu(0)$, that is

$$
j(D)=\frac{j_{0}}{1-j_{0} \ln \frac{D_{0}}{D}} .
$$

Scaling can be performed down to $D \sim k_{B} T$, also we see that, if $j_{0} \equiv j\left(D_{0}\right)>0$ (antiferromagnetic coupling), the running coupling constant $j$ increases. Eq. (38) shows that $D e^{-\frac{1}{j}}$ is a constant of this flow, which defines an energy scale as $T_{K}$ :

$$
k_{B} T_{K}=D_{0} e^{-\frac{1}{j_{0}}}
$$

In general, at the Kondo temperature $T_{K}$ the system has approached the scale at which the perturbative analysis breaks down, so that for $T<T_{K} j$ starts to diverge in the flow.

Now we discuss the conductance in such a perturbative limit. Let us take a $\delta(x)$-like dot with an even barrier potential $\left(\Gamma_{R}=\Gamma_{L}\right)$. To lowest perturbative order the leading self-energy correction to $t^{0}\left(\omega+i 0^{+}\right)$(see eqs. (16), (17)) $(\omega=0)$ yields:

$$
g=\left|\frac{\pi}{k_{o}} t^{0}\right|^{2}=\Re e\left\{\frac{i \pi}{k_{o}} t^{0}\right\} \approx \pi^{2}(\nu(0) J)^{2}
$$

where $\frac{1}{\nu(0) J}=\ln \frac{T}{T_{K}}$ is the invariant charge defined through eq. (40), so that the conductance can be written as:

$$
g \approx \pi^{2} \ln ^{-2} \frac{T}{T_{K}}, \quad T_{K} \ll T \ll D .
$$

The tail of the conductance in the perturbative limit $T \gg T_{K}$ is logarithmic.

\section{One channel anisotropic Kondo model and the Toulouse limit}

In the following we rewrite the Coulomb gas approach introduced in subsection $V . B$ focusing on the single channel anisotropic Kondo system. Let us start from the general effective Hamiltonian:

$$
H_{\text {eff }}^{\mathrm{A}}=H_{\text {lead }}+H_{K}^{\mathrm{A}} \equiv H_{\text {lead }}+J_{z} S_{d}^{z} \sigma_{z}(0)+\frac{J_{\perp}}{2}\left(S_{d}^{+} \sigma_{-}(0)+S_{d}^{-} \sigma_{+}(0)\right) .
$$

As stated in ref. [21], the perturbation term (the one containing $J_{\perp}$ ) has the effect of flipping the local spin at each application; hence the problem of calculating the partition function of such a system reduces to the evaluation of the amplitude for a succession of spin flips at times $\tau_{1}, \tau_{2}, \ldots$ and the Feynman sum over histories is the sum over all possible numbers and positions of flips. So, we get the expression (29) which can be rewritten as:

$$
Z_{C G}=\sum_{N=0}^{\infty}\left(J_{\perp} \tau_{M}\right)^{2 N} \int_{0}^{\beta} \frac{d \tau_{2 N}}{\tau_{M}} \ldots \int_{0}^{\tau_{2}-\tau_{M}} \frac{d \tau_{1}}{\tau_{M}}\left[e^{+\sum_{i \neq j=1}^{2 N}(-1)^{i+j}(2-\varepsilon) \ln \left|\frac{\tau_{i}-\tau_{j}}{\tau_{M}}\right|}\right]
$$

Here $\varepsilon$ is the quantity:

$$
\varepsilon=\frac{8 \delta_{A F}}{\pi}-\frac{8 \delta_{A F}^{2}}{\pi^{2}} \simeq 2 J_{z} \tau_{M}
$$

where $\delta_{A F}$ is the scattering phase shift of antiferromagnetic sign introduced by the $J_{z} S_{d}^{z} \sigma_{z}(0)$ term. It can be seen clearly that the sign of $J_{z}$, or $\varepsilon$, determines whether the coupling is ferromagnetic or antiferromagnetic, so the condition $\varepsilon>0$ gives the antiferromagnetic coupling.

Let us now notice that eq. (44) is a function of three parameters only: $\frac{\tau}{\tau_{M}}, J_{\perp} \tau_{M}, \varepsilon$; we are interested to the case $\tau \rightarrow \infty$ (low temperature). In such a case it is well known that the ferromagnetic Kondo system has a mean spin moment which corresponds to a long range order of the classical system: the positive and negative charges are all 
bound in pairs forming dipoles all pointing in the same direction. The phase transition occurs, at least for small $J_{\perp}$, at the ferromagnetic-antiferromagnetic boundary point $\varepsilon=0$.

For such a system it is possible to derive a set of scaling equations by renormalization of the cut-off $\tau_{M}$; these laws are exact for small $J_{\perp} \tau_{M}$ and $\varepsilon$. The physical picture is that of many close pairs of flips which change slightly the mean magnetization, located between pairs of isolated flips which are reversals of the magnetization over a larger timescale; so, the isolated flips can be considered as acting in a medium where the close pairs modify the mean magnetization. This line of reasoning leads to the following scaling laws for the "fugacity" $J_{\perp} \tau_{M}$ and the "charge" $\varepsilon$ respectively:

$$
\frac{d\left(J_{\perp} \tau_{M}\right)}{d \ln \tau_{M}}=\frac{\varepsilon}{2}\left(J_{\perp} \tau_{M}\right) ; \quad \frac{d \varepsilon}{d \ln \tau_{M}}=(2-\varepsilon)\left(J_{\perp} \tau_{M}\right)^{2} .
$$

Let us make a few comments on such equations. First, these are also exact for finite $\varepsilon$, because only $J_{\perp} \tau_{M}$ need to be small. Second, they are compatible in the isotropic case $J_{z}=J_{\perp}, \varepsilon \simeq 2 J_{z} \tau_{M} \simeq 2 J_{\perp} \tau_{M}$ where $J_{\perp} \tau_{M}$ and $\varepsilon$ are small; because $J_{\perp} \tau_{M}$ and $\varepsilon$ scale together, the isotropic Kondo system remains isotropic at every time scale. In the anisotropic model, eqs. (46) become:

$$
\frac{d \varepsilon}{d\left(J_{\perp} \tau_{M}\right)} \simeq 4 \frac{J_{\perp} \tau_{M}}{\varepsilon} ; \varepsilon^{2}-4 J_{\perp}^{2} \tau_{M}^{2}=\text { const }
$$

thus the scaling lines are a set of hyperbolas with asymptotes corresponding to the isotropic case.

We see clearly from Fig. 3 that all ferromagnetic cases below the isotropic one scale onto the case $J_{\perp} \tau_{M}=0$ which is an ordered one, so the transition line coincide with the ferromagnetic case. Conversely, in the antiferromagnetic (AF) case $J_{\perp} \tau_{M}$ and $\varepsilon$ increase starting from some values, small at will, and we always can find a timescale for which $\varepsilon \sim 1$. Such a timescale is a crucial one for the Kondo phenomenon, it is the Kondo temperature already introduced in the previous sections and sets the scale at which the system behaves as it were strongly coupled. Thus, the renormalization procedure is valid up to $\varepsilon \sim 1$.

Now, let us briefly focus on the so-called Toulouse limit $J_{z}=J_{\perp}, \varepsilon \sim 1$; in such a case the system is equivalent to a simple quadratic model with Hamiltonian:

$$
H_{\mathrm{T}}=\sum_{k} \epsilon_{k} c_{k}^{\dagger} c_{k}+V \sum_{k}\left[d^{\dagger} c_{k}+c_{k}^{\dagger} d\right] .
$$

Here $c_{k}, c_{k}^{\dagger}$ are Fermi operators for free spinless electrons and $d, d^{\dagger}$ are Fermi operators for a local resonant state. The partition function corresponding to the Hamiltonian (48) is exactly equal to the one in eq. (44) for the case of classical charges \pm 1 . Such a theory corresponds to free particles and there is no renormalization.

\section{BREAKDOWN OF PERTURBATIVE APPROACH AT $T \sim T_{K}$ : STRONG-COUPLING FIXED POINT}

In the previous Section we have derived the perturbative flow equation for the Kondo coupling constant $J$. Integration of the renormalization group (RG) equation shows that $J$ grows as $T$ is lowered, until the perturbative analysis does not make any sense anymore. The question whether the RG flow stops at some finite- $T$ scale fixed coupling or goes all the way down to $T=0$ has been widely debated in the literature (see [5] for a review on the subject). However, from numerical RG analysis and from the exact Bethe-ansatz solution of the model [7] it is now clear that the system takes no fixed points at finite $T$, but the RG flow goes all the way down to $T=0$. So, the aim of this Section is to discuss the physics of the Kondo system in the $T=0$-unitarity limit.

In the case of a localized spin-1/2 impurity antiferromagnetically interacting with the spin of one type of itinerant electrons only (spin-1/2, one channel Kondo effect), the theory of the unitary limit was first developed by Noziéres [40]. As $T$ is lowered all the way down to 0 , the flow of the coupling strength runs all the way toward an $\infty$-coupling fixed point. At the fixed point, the impurity spin is fully screened and the localized magnetic moment is effectively substituted by a spinless potential scattering center with infinite strength (which forbids double occupancy at the impurity site). The $T \rightarrow 0$ "unitarity limit" is well-described by a Fermi liquid theory, which also allows for calculation of finite- $T$ corrections to fixed-point values of the physical quantities. Both elastic and inelastic scattering processes provide finite- $T$ contributions to the conductance. As we will show in the next section, the two kinds of processes contribute at the same order, thus giving raise to corrections to the unitary limit proportional to $\left(\frac{T}{T_{K}}\right)^{2}[40]$.

A generalization of Kondo's original idea was done by Noziéres and Blandin [41]. They showed that interaction of itinerant electrons with magnetic impurities in metals may involve electrons with different quantum numbers besides 
the spin: for instance, electrons with different orbital angular momentum. Different orbital quantum numbers define different "channels" of interaction. Therefore, "many channel" Kondo effect may arise. Although in the perturbative region there is no qualitative difference between one-channel and many-channel effect, deep differences may arise in the unitarity limit, depending on the number of channels $K$ and on the total spin of the impurity, $S$.

In the case $2 S=K$, at $T=0$ the magnetic moment at the impurity is fully screened by electrons from the leads. The impurity forms a singlet state with $2 S$ conduction electrons and no other electrons can access the impurity site. The system behaves exactly as if there were no impurity, besides a boundary condition on the wavefunction of conduction electrons which takes into account that the impurity site is "forbidden" to them [40]. Such a Fermi liquid state is stable against leading finite- $T$ corrections, as we shall see below, and corresponds to an infinite effective coupling $\nu(0) J$. A special case of this is the one channel Kondo just discussed, with $S=\frac{1}{2}$ and $K=1$.

In the case $2 S>K$, in the strong coupling regime a residual magnetic moment is still present at the impurity, since there are no more conduction electrons able to screen the localized spin. The corresponding fixed point is again a Fermi liquid, but with a localized partially screened magnetic moment at the impurity that is ferromagnetically coupled to the itinerant electrons: it again provides the stability of the local Fermi liquid.

A very special case is the $2 S<K$ one. In such a case conduction electrons attempt to "overscreen" the impurity in the strong coupling limit, that is the resulting magnetic moment is opposite to the original one. The coupling among the localized residual magnetic moment and the itinerant electrons is now antiferromagnetic. It drives the system out of the strongly coupled regime towards a finite-coupling fixed point $J_{*}$ [41], as we clearly see in Fig. 4. At $J=J_{*}$ the leading finite- $T$ interaction is given by a marginal three-particle operator which breaks down the Fermi liquid state and generates a non-Fermi liquid behavior in the physical quantities. We will discuss such an issue in the Section VII of this review.

\section{THE FULLY SCREENED SINGLE CHANNEL KONDO CASE}

\section{A. Finite temperature corrections to the one-channel Kondo conductance}

At $T=0$ the linear response of the Kondo system to an applied voltage bias reaches the so called unitarity limit.

The response function is the resistivity in a bulk system (magnetic impurities in diluted alloys), while it is the conductance in a quasi one-dimensional system as the system of our interest: a dot with applied contacts.

The striking feature of the Kondo effect in diluted alloys is the minimum in the resistivity at low temperature which violates the expected property: $d \rho / d T>0$. Indeed, well below $T_{K}$ the resistivity increases again up to a maximum value proportional to the number of impurities $N_{i}$ per unit volume ("unitarity limit").

On the contrary a maximum of the conductance is expected at $T=0$ for Kondo conductance across a quantum dot, where the unitarity limit that is reached is $\frac{2 e^{2}}{h} g_{u}$.

Being the $s$-wave scattering effectively one-dimensional like, such a 'reversed behavior' looks paradoxical. However, it is just a consequence of the difference between the 3-d and 1-d impurity scattering, as we explain here. In order to illustrate the difference, it is enough to note that both facts stem from the main feature of Kondo impurity scattering: the formation of a resonance at the Fermi level due to many-body effects, which implies that the phase shift reaches the value $\delta=\pi / 2$ and that scattering is resonant at the impurity. This produces different results in the two cases:

3-d: a spherical $s$ - wave is diffracted from the impurity with maximum amplitude at the resonance, what increases the flux propagating backward and enhances resistivity.

1-d : resonant scattering coincides with resonant trasmission in this case, what implies the vanishing of backward reflection and enhances the conductance.

Temperature corrections are twofold. One is due to the energy dependence of the phase shift close to the resonance $\delta^{0}(\epsilon)=\pi / 2-a \epsilon^{2}$ and to the fact that energies close to the Fermi surface are sampled, because the Fermi functions are not step-like at finite $T$. The second one is due to inelastic processes which produce transitions from the singlet ground state to the excited states. The latter can be accounted for with an expansion in inverse powers of the singlet binding energy [40]. We include a simplified approach to the problem which rests on the Fermi liquid nature of the excitation spectrum in Appendix B [40] [8] [10]. It is found that corrections to the conductance are quadratic in temperature, as it is usual in the Fermi liquid theory:

$$
g=g_{u}\left[1-\left(\frac{\pi T}{T_{K}}\right)^{2}\right], \quad T \ll T_{K} .
$$


The weak-coupling $\left(T \gg T_{K}\right)$ and the strong-coupling $\left(T \ll T_{K}\right)$ asymptotes of the conductance, eqs. (42) and (16), have a very different structure but, since the Kondo effect is a crossover phenomenon rather than a phase transition [6] [7], the dependence $g(T)$ is a smooth function [10] throughout the crossover region $T \sim T_{K}$ :

$$
g=g_{u} f\left(\frac{T}{T_{K}}\right)
$$

The universal function $f(x)$, as found by resorting to numerical renormalization group (NRG) in refs. [10] [11], is plotted in Fig. 5. It interpolates between $f(x \gg 1)=\frac{3 \pi^{2}}{16}(\ln x)^{-2}$ and $f(0)=1$.

\section{B. The Kondo resonance in a magnetic field}

A small magnetic field $B$ lifts the degeneracy of the spin states at the impurity.This produces a splitting of the resonance and a change of its shape which has been numerically studied mostly via NRG [11]. The splitting of the peak increases linearly with $B$ and is $\Delta=2 \mu_{B} B$, i.e. twice the Zeeman spin splitting.

This can be understood easily if one considers the particle and hole virtual occupations which mediate the Kondo interaction.

Let us consider the symmetric case $\epsilon_{d}=-U / 2$. The dot states with $N$ electrons and one unpaired spin are $N \uparrow$ and $N \downarrow$ corresponding to $\epsilon_{d}+B / 2\left(\mu_{B}=1\right)$ and $\epsilon_{d}-B / 2$, respectively. In presence of spin splitting two particle processes are allowed: $p_{\sigma}\left(p_{-\sigma}\right)$ in which a spin $-\sigma$ is added to the state $N \sigma$ and subsequently a spin $-\sigma$ is removed with the energy balance:

$p_{\downarrow} \rightarrow \delta E(N \uparrow,+\downarrow)+\delta E(N+1,-\uparrow)=U+(-U-B)=-B$
$p_{\uparrow} \rightarrow \delta E(N \downarrow,+\uparrow)+\delta E(N+1,-\downarrow)=(U+B)+(-U)=B$.

Similarly the hole processes have an energy balance:

$h_{\uparrow} \rightarrow \delta E(N \uparrow,-\uparrow)+\delta E(N-1,+\downarrow)=\left(-\epsilon_{d}-B / 2\right)+\left(\epsilon_{d}-B / 2\right)=-B$

$h_{\downarrow} \rightarrow \delta E(N \downarrow,-\downarrow)+\delta E(N-1,+\uparrow)=\left(-\epsilon_{d}+B / 2\right)+\left(\epsilon_{d}+B / 2\right)=B$.

This implies that there are two peaks in the spectral density at $\omega= \pm B$ corresponding to the $p_{\downarrow}, h_{\uparrow}$ and $p_{\uparrow}, h_{\downarrow}$ spin flip processes, respectively.

The value at the Fermi energy of the spectral function is related to the $B$-dependent phase shift in the Fermi liquid picture by the Friedel sum rule:

$$
-\pi \nu(0) \Im m\left(t_{\sigma}(\omega=0, T=0, B)\right)=\sin ^{2} \delta_{\sigma}(B) .
$$

The Bethe Ansatz solution of the problem relates the phase shift at the Fermi level to the magnetization of the impurity: $\delta_{\sigma}(B)=\frac{\pi}{2}\left[1-2 M_{d}(B)\right]$ [43]. Hence a reduction of the peak height with increasing of the magnetic field follows.

It has also been argued that the renormalizability of the Kondo problem could break down when $B$ exceeds some critical value related to the Kondo temperature, because of the back action of the induced conduction electron polarization cloud on the impurity leading to a broken symmetry state with $<S_{z}>\neq 0[44]$.

\section{Crossing of the dot levels in a magnetic field and enhancement of the Kondo conductance}

Conventional Kondo resonant transmission requires a magnetic moment to be present on the dot. Usually dots with an even number of electrons are in a singlet state, while dots with an odd number of electrons have an unpaired spin and have a doublet GS. Hence, there is a parity effect: CB conduction valleys with $N=$ even do not display the Kondo conductance while those with $N=$ odd do. An exception to this rule occurs at zero magnetic field when Hund's rule applies. This was found experimentally in vertical QD e.g. at $N=6$. The GS of the isolated dot has $S=1$ (triplet) [45]. An underscreened Kondo effect is expected at this point and the GS of the interacting system becomes a doublet. By applying a weak magnetic field orthogonal to the dot $(B=0.22 T e s l a)$, a transition of the GS from triplet to singlet (T-S) has been found. The single particle energy levels become angular momentum dependent and Hund's rule no longer applies. Close to the crossing a remarkable enhancement of Kondo coupling with increase of the Kondo temperature was experimentally found. Indeed, scaling shows a non universal critical temperature when the interplay of the fourfold degenerate states $\left(\mid S S_{z}>\right.$ with $\left.S=0,1\right)$ is included [46].

An extended and unified approach to the problem can be found in [47]. A minor difference is the fact that they consider an in-plane magnetic field as the source of the crossing which could produce Zeeman spin splitting (ZSS) of 
the single particle states. The four dot states are mapped onto a two impurities Kondo model (2IKM) [48] with spin $\vec{S}_{1}, \vec{S}_{2}$. However they are coupled by a potential term $V n \rho_{n n}(0) \vec{S}_{1} \cdot \vec{S}_{2}$ and an exchange term $i \operatorname{Ins} s_{\bar{n} n}(0) \cdot \vec{S}_{1} \times \vec{S}_{2}$. Here $\rho, \sigma$ are the charge and spin density of the conduction electrons with $n$ and $\bar{n} \neq n$ labeling two different orbital states. Because these terms violate the invariance under particle-hole transformation, the 2IKM cannot flow by scaling to the non Fermi-liquid fixed point, which is known to be a remarkable feature of the model. In the case of large ZSS $\Delta$ the RG flow terminates at $D \approx \Delta$. Two of the four states are ruled out in the flow and conduction electrons couple to one single effective spin $1 / 2$ with one extra unusual term in the effective Hamiltonian which is a Zeeman term for the conduction electrons.

Kondo conductance can take place in a dot with an even number of electrons also in a strong vertical magnetic field [35]. Orbital effects induced by B can produce the reversed transition from the singlet to the triplet state (S-T). Indeed, a vertical magnetic field on an isolated dot favors transitions to higher spin states [49]. In this case the ZSS is anyhow sizeable and the crossing involves the singlet state and the component of the triplet state lowest lying in energy. In a vertical geometry with cylindrical symmetry orbital angular momentum $m$ and $z$ - spin component $\sigma$ are good quantum numbers. In particular because the singlet state has total angular momentum $M=0$ and the triplet state involved in the crossing has $M=1$, only a $(m=0 \downarrow)$ electron can enter the dot when it is in the triplet state. On the contrary, only an $(m=1 \uparrow)$ electron can enter the dot, if it is in the singlet state. This implies that there is one single channel of conduction electrons involved in cotunneling processes, with orbital and spin degrees of freedom locked together. Again, a residual effective spin $1 / 2$ survives at the dot, while $N$ is even [23]. This is another striking manifestation of the spin-charge separation that occurs at the Kondo fixed point. Usual Kondo coupling leads to $N=$ odd together with $S=0$. In the complementary situation here described, it is $N=$ even and $S=1 / 2$.

\section{THE OVERSCREENED TWO CHANNEL KONDO CASE}

A two channel Kondo behavior has been invoked in an experiment by Ralph and Buhrman [50] on clean Cu point contacts, defects in the metal that can be described by two level systems (TLS). The TLS could tunnel between the even and odd state with the assistance of the conduction electrons. Their physical spin is not involved in the scattering, so that two channels are available [51]. It is still unclear whether the Kondo temperature can be large enough so that any effect of the Kondo correlation can be measured [52]. However, these experiments have triggered renewed interest in two channel Kondo conductance. The temperature and voltage dependence of the conductivity have been numerically calculated within the "Non Crossing Approximation" (NCA) [53] and found to be consistent with a scaling Ansatz motivated by the Conformal Field Theory (CFT) solution of the problem [8]. Since then, no other experimental proof of two channel Kondo effect in impurities has been produced. The result for the imaginary part of the transmission $t$ is:

$$
\Im m t(\omega) \sim \sqrt{\frac{\omega}{T_{K}}} .
$$

From this result, we get a $\sqrt{T / T_{K}}$ temperature dependence of the conductance as $T \rightarrow 0$, which is a clear signature of the breakdown of the Fermi liquid [8] (see Fig. 6).

In order to emphasize the deep difference between single-channel and many-channel Kondo effect in the $T=0$ limit we just mention here that the imaginary part of the proper self-energy, close to the Fermi surface, behaves as $\Im m \Sigma(k, \omega) \propto C_{k} \omega^{2}$ (where the chemical potential $\mu=0$ is taken as reference energy) in the Fermi liquid case

and in the single channel Kondo problem. On the contrary, it behaves as $\varsigma m \Sigma(k, \omega) \propto C_{k}^{\prime} \omega^{\frac{1}{2}}$ in the two channel "overscreened" Kondo problem. In the following we refer, for simplicity, to the two channel "overscreened" case. Through the Kramers-Kronig relation

$$
\frac{\partial}{\partial \omega} \Re e \Sigma(k, \omega)=-\frac{1}{\pi} P \int_{-\infty}^{\infty} d \omega^{\prime} \frac{\partial_{\omega} \Im m \Sigma\left(k, \omega^{\prime}\right)}{\omega^{\prime}-\omega}
$$

we see that $\left.\frac{\partial}{\partial \omega} \Re e \Sigma(k, \omega)\right|_{\omega \rightarrow 0}$ is finite in the first case, at the Fermi surface, on the contrary it has a power law divergence in the second case. It follows that the quasiparticle pole residue $z_{k}=\left.\left[1-\partial_{\omega} \Sigma(k, \omega)\right]^{-1}\right|_{\omega \rightarrow 0}$ vanishes as a power law in the second case at the Fermi surface. Luttinger [54] showed that, to all orders of perturbation theory in the interaction, the imaginary part of the proper self-energy behaves as $\varsigma m \Sigma(k, \omega) \propto C_{k} \omega^{2}\left(C_{k}>0\right)$ close to the Fermi surface, which implies an infinite lifetime for the quasiparticles at the Fermi surface. The Fermi surface is sharp and well defined. These are the foundation stones of the normal Fermi liquid Theory and they are invalidated in the spin- $\frac{1}{2}$ two channel Kondo case. 
In this Section we focus, in particular, on two channel spin-1/2 Kondo effect in both the perturbative region and the unitarity limit. We describe the scaling perturbative approach and the bosonization $(T \sim 0)$ technique, respectively. An attempt to extend the Anderson-Yuval approach of Section $I V . C$ to the two channel Kondo case can be found in $[42]$.

\section{A. Perturbative analysis at $T>>T_{K}$}

The starting point of our reasoning is the effective Hamiltonian given in eq. (35), which we will take in the isotropic limit. In the following we will restrict our analysis to a two-fold degenerate dot level. In this case, as we pointed out, the QD can be modelized as a spin-1/2 magnetic impurity, whose spin is given by:

$$
S_{d}^{a}=\frac{1}{2} d_{\gamma}^{\dagger} \tau_{\gamma \gamma^{\prime}}^{a} d_{\gamma^{\prime}}
$$

(that $\vec{S}_{d}$ is a spin-1/2 comes out from the identity $\vec{S}_{d}^{2}=3 / 4$, valid in the case of single-occupancy for the dot's level).

In this case and for a generic number of channels for the itinerant electrons, eq. (35) takes the form:

$$
H_{K}=J \sum_{a}\left(\sum_{\gamma \gamma^{\prime}}\left(d_{\gamma}^{\dagger} \frac{\tau_{\gamma \gamma^{\prime}}^{a}}{2} d_{\gamma^{\prime}}\right) \sum_{k k^{\prime}} \sum_{\sigma \sigma^{\prime} ; \alpha}\left(c_{k \sigma \alpha}^{\dagger} \frac{\tau_{\sigma \sigma^{\prime}}^{a}}{2} c_{k^{\prime} \sigma^{\prime} \alpha}\right)\right)
$$

where $\alpha \in(1, \ldots, K)$ is the channel index and the constant $J$ is taken as a perturbative parameter $(>0)$. Infrared divergent diagrams provide a flow of $J$ as a function of $T$. We are now going to derive the perturbative $\beta$-function at third order in $J$. At finite $T$ the Green function in Fourier space will depend on the momentum of the particles and on the Matsubara frequencies $\omega_{m}=\frac{2 \pi}{\beta}\left(m+\frac{1}{2}\right)$ (for fermions). In our case, the Green function for the lead electrons is given by:

$$
G_{\sigma \sigma^{\prime} ; \alpha \alpha^{\prime}}\left(i \omega_{m}, k\right)=\mathbf{F T}\left\{\left\langle\hat{T}\left[c_{\sigma \alpha}(\tau, k) c_{\sigma^{\prime} \alpha^{\prime}}^{\dagger}(0, k)\right]\right\rangle\right\}=\frac{\delta_{\sigma \sigma^{\prime}} \delta_{\alpha \alpha^{\prime}}}{i \omega_{m}-v_{F} k}
$$

where FT stands for 'Fourier transform' and $\hat{T}$ is the time ordering operator, while the Green function for the $d$-fermion is:

$$
\mathcal{G}_{\gamma \gamma^{\prime}}\left(i \omega_{m}\right)=\mathbf{F T}\left\{\left\langle\hat{T}\left[d_{\gamma}(\tau) d_{\gamma^{\prime}}^{\dagger}(0)\right]\right\rangle\right\}=\frac{\delta_{\gamma \gamma^{\prime}}}{i \omega_{m}}
$$

The interaction vertex determined by $H_{\text {eff }}$ is:

$$
V_{\sigma \sigma^{\prime} ; \gamma \gamma^{\prime}}^{\alpha \alpha^{\prime}}\left(\left\{i \omega_{m}^{(j)}\right\}\right)=\delta_{\alpha \alpha^{\prime}} \frac{J}{4} \tau_{\gamma \gamma^{\prime}}^{a} \tau_{\sigma \sigma^{\prime}}^{a} \delta\left(\omega_{m}^{(1)}+\omega_{m}^{(2)}-\omega_{m}^{(3)}-\omega_{m}^{(4)}\right)
$$

The one-loop structure of the theory provides a renormalization to the interaction vertex, that is, to the coupling constant, by means of the two diagrams drawn in Fig. 7. The sums over Matsubara frequencies can be calculated by using the standard techniques described, for example, in [55]. In the low-energy limit for the electrons from the leads (that is, if only excitations about the Fermi level are taken into account), the sum of the two diagrams is given by:

$$
\mathcal{D}_{1}+\mathcal{D}_{2} \approx-i \frac{J^{2}}{8} \delta_{\gamma \gamma^{\prime}}\left\{\left[\delta_{\sigma \sigma^{\prime}} \delta_{\alpha \alpha^{\prime}}+\tau_{\sigma \sigma^{\prime}}^{a} \tau_{\alpha \alpha^{\prime}}^{a}\right]+\left[-\delta_{\sigma \sigma^{\prime}} \delta_{\alpha \alpha^{\prime}}+\tau_{\sigma \sigma^{\prime}}^{a} \tau_{\alpha \alpha^{\prime}}^{a}\right]\right\} 2 \nu(0) \ln \left(\frac{D}{k_{B} T}\right)
$$

( $D$ is an ultraviolet cutoff, identified with the width of the conduction band). The corresponding renormalization to the coupling constant $J$ is easily worked out and is given by:

$$
\Delta^{(2)} J(T, D)=2 \nu(0) J^{2} \ln \left(\frac{D}{k_{B} T}\right)
$$

A careful analysis of the vertex renormalization at third order in $J$ reveals that several third-order diagrams are already taken into account by the scaling equation generated by the second-order vertex correction, as discussed in [56]. The only "new" contribution comes from the "non-parquet" diagram shown in Fig. 8. Because of the loop over 
the fermions from the contacts, such a contribution carries an overall factor of $K$, that is, the diagram is proportional to the number of channels and the correction to the coupling constant up to third-order in $J$ is:

$$
\Delta^{(3)} J=\left(2 \nu(0) J^{2}-2 K(\nu(0))^{2} J^{3}\right) \ln \left(\frac{D}{k_{B} T}\right) .
$$

Integration of the renormalization group equation for $J$ provides:

$$
-\frac{1}{2 j}+\frac{1}{2 j_{0}}+\frac{K}{2} \ln \left[\frac{j}{j_{0}}\left(\frac{1-K j_{0}}{1-K j}\right)\right]=x-x_{0}
$$

where $j=\nu(0) J$ and $x=\ln \left(\frac{D}{k_{B} T}\right)$.

It is seen from eq. (61) that the fixed point is reached at an intermediate coupling $j^{*}$. However, the perturbative RG analysis is not conclusive here, because even in the ordinary, single channel, Kondo model an artificial intermediate coupling fixed point is produced when the perturbative RG equations are expanded to 3rd order [56].

It is straightforward to infer the Kondo temperature $T_{K}$ from eq. (61). Usually $T_{K}$ is defined as the temperature scale at which $j$ becomes $\mathcal{O}(1)$. Following such a criterion, at $T=T_{K}$ we can neglect $1 / j$ compared to $1 / j_{0}$ and obtain the following approximate formula for $T_{K}$ :

$$
k_{B} T_{K} \approx D\left(j_{0}\right)^{\frac{K}{2}} e^{-\frac{1}{2 j_{0}}} .
$$

Eq. (62) is quite general, in that it provides the value for the crossover temperature for any number of channels $K$. This proves that the way the system approaches the scale at which the perturbative analysis breaks down does not depend on the number of channels, except for a redefinition of the Kondo temperature. Hence, logarithmic divergencies are expected when approaching to the $T_{K}$, no matter on what the number of channels is. In the next subsection we will analyze the $T=0$ behavior of such a system, and will see that it is dramatically different from the one channel case for what concerns the fixed-point properties.

\section{B. Analysis at $T \sim 0$}

Several techniques have been applied in order to get informations about physical quantities around the fixed point in the Kondo regime in such a limit. Finite- $T$ corrections have been derived by means of bosonization techniques [57] [58], of Bethe-ansatz like exact solutions [7] and of Conformal Field Theory (CFT) techniques [8]. Now, CFT approach is extremely effective in calculating finite- $T$ corrections, Wilson ratios and other exact results concerning Green functions [8], but its starting point that charge, spin and "flavor" quantum numbers are enough to identify a primary fermionic field in the theory leads to an inconsistency: the corresponding on-shell $S$ matrix comes out to be 0. The solution to such an "unitarity paradox" has been suggested by Ludwig and Maldacena [59], who introduced a fourth "spin-flavor" quantum number. So, while at the unitarity limit charge, spin and flavor do not change upon scattering off the impurity, the spin-flavor changes, giving rise to one more scattering channel. Such an extra quantum number allows for an off-diagonal on-shell $S$ matrix, therefore the unitarity paradox simply means that the diagonal elements of the $S$ matrix with respect to the spin-flavor number are 0. However, a theory of the unitarity limit, needed in order to compute, for instance, within an unified framework transport properties with finite- $T$ corrections, is not yet fully developed. In this subsection we briefly sketch the first steps in order to derive an appropriate scattering potential a' la Noziéres, in the case of two channel spin- $1 / 2$ overscreened Kondo effect. We follow an approach equivalent to the one by Tsvelick [60], that is the introduction of a regularization procedure able to move the fixed point toward infinite-coupling. Then, we go through a bosonization - refermionization in order to account for the scattering processes with changing of the spin-flavor. The bosonization procedure allows us to split the degrees of freedom involved in our problem: in this way we will show that the Kondo interaction involves only the spin and spin-flavor degrees of freedom while the charge and flavor ones are fully decoupled. This remark is a crucial one because it makes possible to derive a $S$ matrix in the unitarity limit which comes out to be diagonal in any quantum number, but the spin-flavor; such a $S$ matrix describes the whole system of dot and contacts.

In the following we discuss the case $K=2, S=1 / 2$, so we have two flavors of conduction electrons from an effectively one-dimensional conductor which interact with a localized spin-1/2 magnetic impurity.

Let $c_{\alpha \sigma}(x)$ be the lead electron operators $(\sigma=\uparrow, \downarrow$ is the spin index, $\alpha=1,2$ is the flavor index). A lattice-model complete Hamiltonian is written as: 


$$
\begin{aligned}
H^{2 C h}= & -t \sum_{x}\left[c_{\alpha \sigma}^{\dagger}(x) c_{\alpha \sigma}(x+a)+c_{\alpha \sigma}^{\dagger}(x+a) c_{\alpha \sigma}(x)\right] \\
& -\mu \sum_{x} c_{\alpha \sigma}^{\dagger}(x) c_{\alpha \sigma}(x)+J \vec{S}_{d \cdot} \cdot\left[\vec{\sigma}_{1}(0)+\vec{\sigma}_{2}(0)\right]
\end{aligned}
$$

where $\vec{\sigma}_{\alpha}(x)=\frac{1}{2} c_{\alpha \sigma}^{\dagger}(x) \vec{\tau}_{\sigma \sigma^{\prime}} c_{\alpha \sigma^{\prime}}(x)$ and $a$ is the lattice spacing.

Now, we can linearize the dispersion relation around the Fermi surface and introduce two chiral fields $c_{ \pm, \alpha \sigma}(x)$. Even and odd parities can be introduced to obtain fields with the same chirality, $\phi_{\alpha \sigma}^{e}$ and $\phi_{\alpha \sigma}^{o}$, so odd parity fully decouples from the interaction Hamiltonian. In order to properly deal with the interacting fields, we will bosonize $\phi_{\alpha \sigma}^{e}$; in particular, we define four bosonic fields $\Psi_{\alpha \sigma}$ in terms of which it is possible to express densities of the remarkable physical quantities. To this end we are led to construct the four bosonic fields $\Psi_{X}(X=\mathrm{ch}, \mathrm{sp}, \mathrm{fl}, \mathrm{sf})$ for the charge, spin, flavor and spin-flavor degrees of freedom [58] [59] [61] as linear combinations of the previous ones.

In this way it is possible to realize two "inequivalent" representations of the fields $\phi_{\alpha \sigma}^{e}$ in terms of the fields $\Psi_{X}$ $(X=\mathrm{ch}, \mathrm{sp}, \mathrm{fl}, \mathrm{sf}), \phi_{\alpha \sigma ; I}^{e}$ and $\phi_{\alpha \sigma ; I I}^{e}$, given by:

$$
\begin{aligned}
\phi_{\alpha \sigma ; I}^{e}(x) & =\eta_{\alpha \sigma}: e^{-\frac{i}{2}\left[\Psi_{\mathrm{ch}}+\sigma \Psi_{\mathrm{sp}}+\alpha \Psi_{\mathrm{fl}}+\alpha \sigma \Psi_{\mathrm{sf}}\right](x)}: \\
\phi_{\alpha \sigma ; I I}^{e}(x) & =\xi_{\alpha \sigma}: e^{-\frac{i}{2}\left[\Psi_{\mathrm{ch}}+\sigma \Psi_{\mathrm{sp}}+\alpha \Psi_{\mathrm{fl}}-\alpha \sigma \Psi_{\mathrm{sf}}\right](x)}:
\end{aligned}
$$

where $\eta$ and $\xi$ are suitable Klein factors. Notice that the two fields differ only in the spin-flavor quantum number but such a difference is a crucial one.

Now we are ready to rewrite the two channel Kondo interaction Hamiltonian in bosonic coordinates $\Psi_{X}$ as:

$$
\begin{aligned}
H_{K}^{2 C h} & =J\left\{S_{d}^{+}: e^{-i \Psi_{\mathrm{sp}}(0)}:: \cos \left(\Psi_{\mathrm{sf}}(0)\right):+S_{d}^{-}: e^{i \Psi_{\mathrm{sp}}(0)}:: \cos \left(\Psi_{\mathrm{sf}}(0)\right):+S_{d}^{z} \frac{L}{2 \pi} \frac{d \Psi_{\mathrm{sp}}(0)}{d x}\right\} \\
& =J \vec{S}_{d} \cdot\left[\vec{\Sigma}_{g}(0)+\vec{\Sigma}_{u}(0)\right]
\end{aligned}
$$

where the spin densities $\vec{\Sigma}_{A, B}(x)$ are given by:

$$
\begin{array}{lll}
\Sigma_{g}^{ \pm}(x)=: e^{ \pm i\left[\Psi_{\mathrm{sp}}+\Psi_{\mathrm{sf}}\right](x)}: & \Sigma_{g}^{z}(x)=\frac{L}{4 \pi} \frac{d}{d x}\left[\Psi_{\mathrm{sp}}+\Psi_{\mathrm{sf}}\right](x), \\
\Sigma_{u}^{ \pm}(x)=: e^{ \pm i\left[\Psi_{\mathrm{sp}}-\Psi_{\mathrm{sf}}\right](x)}: & \Sigma_{u}^{z}(x)=\frac{L}{4 \pi} \frac{d}{d x}\left[\Psi_{\mathrm{sp}}-\Psi_{\mathrm{sf}}\right](x) .
\end{array}
$$

Both $\vec{\Sigma}_{g}(x)$ and $\vec{\Sigma}_{u}(x)$ are $S U(2)$ spin current operators, so the vector space is made of the bosonic vacuum $|b v a c\rangle$ and the bosonic spin $-\frac{1}{2}$ spinors at a point $x$ :

$$
\begin{aligned}
& \left|\sigma_{g}\right\rangle \equiv: e^{\sigma \frac{i}{2}\left[\Psi_{\mathrm{sp}}+\Psi_{\mathrm{sf}}\right](x)}:|b v a c\rangle \\
& \left|\sigma_{u}\right\rangle \equiv: e^{\sigma \frac{i}{2}\left[\Psi_{\mathrm{sp}}-\Psi_{\mathrm{sf}}\right](x)}:|b v a c\rangle .
\end{aligned}
$$

No triplet adding up $g$ and $u$ spin species together can occur because, given $\vec{\Sigma}_{g, u}=\int_{-L / 2}^{L / 2} d x: \vec{\Sigma}_{g, u}(x)$, we have:

$$
\vec{\Sigma}_{g}\left|\sigma_{u}\right\rangle=0 \quad \vec{\Sigma}_{u}\left|\sigma_{g}\right\rangle=0
$$

that is, if at a point $x$ the spin density associated to $\vec{\Sigma}_{g}$ is $\neq 0$, then, at the same point, the spin density associated to $\vec{\Sigma}_{u}$ is $=0$, and vice versa; this does not allow for overscreening. Such a statement is a crucial one, indeed it corresponds to a particular regularization scheme [60] able to move the finite-coupling fixed point corresponding to the unitarity limit down to $J=+\infty$. At this infinitely-strongly coupled fixed point the impurity spin will be fully screened in a localized spin singlet. In principle, the system might lay within any linear combination mixing the two representations $g, u$, of the form:

$$
\left.|G S\rangle_{\mu}\right|_{x=0}=\left.|\Uparrow\rangle \otimes \frac{1}{2}\left(\left|\downarrow_{g}\right\rangle+\mu\left|\downarrow_{u}\right\rangle\right)\right|_{x=0}-\left.|\Downarrow\rangle \otimes \frac{1}{2}\left(\left|\uparrow_{g}\right\rangle+\mu\left|\uparrow_{u}\right\rangle\right)\right|_{x=0}
$$

where $|\Uparrow\rangle,|\Downarrow\rangle$ are the two impurity states with opposite spin polarizations. Then we search for the two independent linear combinations that do not change upon scattering of lead electrons; it can be shown that such combinations correspond to the values $\mu= \pm i$. 
Now, in the fixed point limit, we "refermionize". Physical states mix both representations, $I$ and $I I$. Scattering by the impurity states should conserve all physical quantum numbers. It can be shown that the elastic scattering in the unitarity limit swaps the two inequivalent representations $I-I I$ of the lead electrons. Correspondingly, the impurity absorbs/emits one spin-flavor quantum. The even parity $S$ matrix has the following representation in the $I-I I$ space:

$$
\mathbf{S}^{0}(\omega=0)=\left(\begin{array}{cc}
0 & -i \\
i & 0
\end{array}\right) \equiv\left(\begin{array}{cc}
0 & e^{-i \frac{\pi}{2}} \\
e^{i \frac{\pi}{2}} & 0
\end{array}\right)
$$

According to eq. (70) the phase shifts induced by the scattering are $\delta^{I I I}(\omega=0)=-\frac{\pi}{4}, \delta^{I I I}(\omega=0)=\frac{\pi}{4}$, while in the case of one channel spin-1/2 Kondo effect the phase shift was $\delta^{0}=\frac{\pi}{2}$. Finally, the conductance can be easily obtained by the Landauer formula using eq. (70) and $\mathbf{S}^{1}=-\mathbf{1}$ :

$$
g=\operatorname{Tr}\{\mathbf{T}\}=\operatorname{Tr}\left\{\left|\frac{1}{2} \sum_{l} S^{l}\right|^{2}\right\}=\frac{1}{2} \operatorname{Tr}\left(\begin{array}{cc}
1 & i \\
-i & 1
\end{array}\right)=1 .
$$

Incidentally, we point out that the groundstate degeneracy always decreases under renormalization, which is the content of "g-theorem" [8]. This leads to a zero temperature entropy for the impurity given by $\mathrm{S}_{i m p}(0)=\frac{1}{2} \ln 2$.

Temperature corrections to this result have also been calculated [60] by using real fermion coordinates (Majorana fermions) $\psi^{a}(x)(a=1,2,3)$, which obey the anticommutation relations $\left\{\psi^{a}(x), \psi^{b}(y)\right\}=\delta^{a b} \delta(x-y)$, describing the relevant coordinates only. This corresponds as well to a regularization scheme where the fixed point has been moved to $J=\infty$. The $\sqrt{T}$ behavior is recovered, as metioned in the introduction to this Section (see eq.(52)).

\section{Can we reach the two channel Kondo fixed point in a quantum dot ?}

A possible experimental realization of the two-channel Kondo fixed point in a quantum dot has been recently proposed [24]: exact diagonalization results of a vertical quantum dot with five electrons show that it can be tuned, by means of a strong external magnetic field, at the degeneracy point between energy levels with $S=1 / 2$, but with different orbital angular momentum. Vertical cylindrical contacts provide single particle energy subbands labeled by the cross-sectional angular momentum and the $k$ vector of the incoming/outgoing electron. Appropriate tuning of the electron density in the contacts offers the chance of including two electron channels only. The advantage of this setting is that no exchange coupling can take place between the channels due to symmetry, so that they act as totally independent. Selection rules due to the cylindrical symmetry enforce angular momentum $m$ conservation and spin component $\sigma$ conservation in the cotunneling processes. For the special setting of the proposed device only a hole $(h)$ process for $\downarrow-$ spin and a particle process for $\uparrow-$ spin are allowed. They differ in the sign of the potential scattering but this difference is inessential. In fact, a particle-hole transformation on the fermion fields of the $\uparrow$ channel only reverses the unwanted sign without affecting the exchange coupling. This puts both channels on an equal footing and points to an "orbital" Kondo coupling where the spin acts as the label for the channel. At this stage the dot plays the role of an effective spin $3 / 2$ impurity, interacting with two channels. So, as it stands the system would flow to an underscreend situation in lowering the temperature. However, provided that Zeeman spin splitting $\Delta$ is larger than the Kondo temperature for the underscreened fixed point $T_{K}^{u}$, there is a crossover temperature $T^{c}$ at which two of the four $S=1 / 2$ states are ruled out of the cotunneling processes, thus allowing for an effective spin $1 / 2$ two channel Kondo flow.

Detecting the effect requires an appropriate control of the hybridization of the dot with the contacts (so that $\Delta>>T_{K}^{u}$ ) and a proper tuning of the gate voltage $V_{g}$. This allows for fine tuning of the exchange couplings between the dot and the two channels. It is well known that the hardest condition to be realized is total equivalence of the channels in the coupling. Were this not the case, the system would prefer one of the two channels as the dominant one and flow to a more conventional one-channel Kondo state. By tuning $V_{g}$ one can cross the point where the two channels are equally coupled which allows for scaling towards the two-channel fixed point. The measured conductance $g(T)$ vs $T$ will exhibit quadratic behavior at low temperature except for a crossover to a square root behavior, at the appropriate $V_{g}$ value which makes the two channels perfectly equivalent (see Fig. 6). The delicate point in this proposal is that the requirement of full cylindrical symmetry of the device is essential.

Another setup for measuring the two channel Kondo conductance has been proposed recently [25]. This proposal relies on an extra contact to provide the second channel. It is recognized however that this would introduce cross exchange terms between the three leads which do not allow for equivalent coupling of the two channels. In fact, any 
diagonalization of the scattering problem of the kind of the one outlined in the previous Subsection to isolate the independent channels can never produce equal coupling as long as off-diagonal terms are non zero. To overcome this difficulty the authors propose that the extra contact is a larger dot itself, having a charging energy $E_{c}$ which hinders exchange coupling with the other two leads. Of course its size should be appropriately tuned. A smaller size implies a larger level spacing $\delta$ inside it and $\delta$ should be low enough because it acts as the low energy cutoff in the scaling toward the fixed point. Therefore, there is a delicate trade-off between incresing $E_{c}$ to prevent cross exchange terms and decreasing $\delta$ not to stop the flow when reducing the temperature.

We have stressed that the NFL fixed point in the two channel Kondo coupling at $T=0$ can only be reached by tuning the exchange couplings of both channels $J_{1}, J_{2}$ exactly equal. Hopefully this can be done by changing an appropriate gate voltage across a critical point $V_{g}^{*}$. According to Fig. 4 the systems flows to $J_{1} \rightarrow \infty, J_{2} \rightarrow 0$ for $V_{g}<V_{g^{*}}$ and to $J_{2} \rightarrow \infty, J_{1} \rightarrow 0$ for $V_{g}>V_{g^{*}}$, both of which are Fermi liquid fixed points. It has been argued that the quantum transition across $V_{g}^{*}$ should display a quantum critical region in the $T-V_{g}$ plane whose critical properties can be determined [26]. This offers better chances to spot whether we are in the vicinity of the NFL fixed point or not, also at finite temperature.

\section{SUMMARY}

In this review we focused on equilibrium transport properties across a quantum dot (QD). A QD is a tunneling center for electrons coming from the leads. Depending on the transparency of the barriers and on the temperature, the coupling $V$ to the leads is weak or strong.

In the weak coupling regime, tunneling can be dealt with perturbatively. In the case of the QD, a Coulomb Blockade zone is delimited by two sharp conduction peaks which grow as $|V|^{2} / T$ in lowering the temperature. Conduction inbetween is due to cotunneling processes and is exponentially damped in temperature. Differential conductance is quite small, being $\propto|V|^{4} / U$, so that the charge degree of freedom is freezed on the dot.

In lowering the temperature, non perturbative coupling of the dot to the delocalized electrons of the contacts can occur and conductance can reach the unitary limit in a CB valley. For pedagogical reasons we have reviewed the old-fashoned Anderson-Yuval model for the correlated state. Otherwise we have used the poor man's scaling approach in the regime where perturbative scaling applies and the Noziéres scattering approach which entails the fixed point physics at zero temperature. These approaches do not allow for quantitative results which are better obtained with the numerical renormalization group (NRG), real time RG and Non Crossing Approximation (NCA) (the last one preferably in the overscreened case when a non Fermi liquid (NFL) fixed point is reached), but they offer a more transparent view of what is going on.

We have briefly reviewed the various types of Kondo couplings.

We have considered the case in which the GS of the dot is degenerate because of spin: if temperature is low enough ( $T<T_{K}$, with $T_{K}$ depending on the transparency of the barriers), spin flip processes proliferate and the magnetic moment of the dot is partially or fully screened. In this case an applied magnetic field has a disruptive effect on the Kondo peak of the conductance. The interesting case of a crossing between different dot spin states induced by the magnetic field has been also discussed.

We have also reported on other possible realizations of Kondo physics involving orbital degrees of freedom (orbital Kondo). The most favourable set up for this case is a vertical geometry of the dot and the contacts with cylindrical symmetry. In this geometry a magnetic field orthogonal to the dot (which may be strong) can induce level crossings and produce the degeneracies of the dot GS which is required for Kondo conductance to take place. Hence one can have Kondo effect also with an even number of electrons on the dot and zero total spin.

Some attention has been devoted to the multichannel Kondo effect. The overscreening case can lead to a NFL fixed point at zero temperature. In particular we have discussed the two channel spin $1 / 2$ Kondo state and reported on possible experimental realizations that have been proposed. It emerges that conditions to be met are very tough. Nonetheless its achievement would probe a beautiful piece of the physics of the strongly correlated systems.

\section{APPENDIX A: RG EQUATIONS FOR THE COULOMB GAS MODEL OF EQ. (29)}

We summarize here the scaling of the action in eq. (29) in order to find out the behaviour of the system at large time scales (low temperature) [21] [34]. The cutoff is rescaled according to: $\tau_{M} \rightarrow(1+\lambda) \tau_{M}$ with $\lambda=\Delta \tau_{M} / \tau_{M} \rightarrow d \ln \tau_{M}$. This adds a factor $e^{-2 N \lambda\left(1-\alpha^{2} / 2\right)}$. The first term arises from $\tau_{M}^{2 N}$ in the denominator, while the one $\propto \alpha^{2}$ arises from 
the $\ln$ - term in the interaction term. Indeed charge neutrality implies that $0=\left(\sum_{i} q_{i}\right)^{2}=\sum_{i \neq j} q_{i} q_{j}+\sum_{i} q_{i}^{2}$ and $q_{i, j}= \pm 1$. This factor renormalizes the fugacity $Y \rightarrow Y+d Y$, with:

$$
Y+d Y \approx Y e^{\lambda\left(1-\alpha^{2} / 2\right)}
$$

what gives the first of eqs. (30). The interaction strength $\alpha$ is renormalized by flip-antiflip (particle-antiparticle) fusion.

Let us consider now all the configurations in which pairs of neighboring charges $q_{i}$ at $\tau_{i}$ and $q_{j}=-q_{i}$ at $\tau_{j}$ (where $j=i \pm 1)$ are at a distance between $\tau_{M}$ and $\tau_{M}(1+\lambda)$. In increasing the scale, these pairs are seen as a neutral compound which screens the interaction between other charges ("fusion " of pairs). Let us consider one single fusion process and develop that part of the action that contains their coordinates:

$$
\begin{aligned}
-S^{(2 N+2)}= & -S^{(2 N)}+q_{i} q_{j} \ln \left|\frac{\epsilon}{\tau_{M}}\right| \\
& +\frac{q_{i}}{2} \sum_{k \neq i, j} q_{k}\left[\ln \left|\frac{\tau_{k}-\tau-\epsilon / 2}{\tau_{M}}\right|-\ln \left|\frac{\tau_{k}-\tau+\epsilon / 2}{\tau_{M}}\right|\right] .
\end{aligned}
$$

Here we have defined $\epsilon / 2=\frac{\tau_{i}-\tau_{j}}{2}$ and $\tau=\frac{\tau_{i}+\tau_{j}}{2}$. The integral over $\tau$ and $\epsilon$ for $\epsilon / \tau_{M}<<1$ which appears in the partition function of eq. (29) is:

$$
\begin{aligned}
& e^{-S^{(2 N)} Y^{2}} \int_{\tau_{i-1}+\tau_{M}}^{\tau_{j+1}-\tau_{M}} \frac{d \tau}{\tau_{M}} \int_{\tau_{M}}^{\tau_{M}(1+\lambda)} \frac{d \epsilon}{\tau_{M}}\left(\frac{\epsilon}{\tau_{M}}\right)^{-\alpha^{2}} \cdot e^{-\alpha^{2} \epsilon \frac{q_{i}}{2} \sum_{k \neq i, j} q_{k} \frac{\partial}{\partial \tau} \ln \left|\frac{\left.\tau_{k}-\tau\right)}{\tau_{M}}\right|} \\
\sim & \lambda Y^{2}\left\{1-\alpha^{2}\left(\frac{q_{i-1}}{2} \sum_{k \neq i, j}^{2 N} q_{k} \ln \left|\frac{\left.\tau_{i-1}+\tau_{M}-\tau_{k}\right)}{\tau_{M}}\right|+\frac{q_{j+1}}{2} \sum_{k \neq i, j}^{2 N} q_{k} \ln \left|\frac{\left.\tau_{j+1}-\tau_{M}-\tau_{k}\right)}{\tau_{M}}\right|\right)\right\},
\end{aligned}
$$

where the term with $k=i-1(k=j+1)$ in the first (second) sum vanishes. Doing the same for each interval $i-j$ and summing, each term is repeated twice. This generates an extra contribution to the action that can be interpreted as the renormalization of the coupling constant:

$$
\alpha^{2} \rightarrow \alpha^{2}+d \alpha^{2} ; d \alpha^{2}=-2 \lambda Y^{2} \alpha^{2} .
$$

Because $\lambda \approx d \ln \tau_{M}$, the second one of eqs. (30) is obtained.

\section{APPENDIX B: TEMPERATURE DEPENDENCE OF THE CONDUCTANCE}

Ohm's law can be derived semiclassically from a Boltzmann equation which accounts for weak scattering of the charge carriers against impurities and defects, when driven by an electric field. Scattering provides a mechanism for relaxation from a non equilibrium to a steady state flow [62]. We follow here a simplified approach starting from the velocity of band electrons $\vec{v}_{k}=\vec{\nabla} \epsilon_{k} / \hbar$. The current density is:

$$
\vec{j}=-\frac{2 e}{3 \mathcal{V}} \sum_{k} \vec{v}_{k}\left(f_{k}-f_{k}^{o}\right)
$$

where $f_{k}\left(f_{k}^{o}\right)$ is the non-equilibrium (equilibrium) Fermi distribution and $\mathcal{V}$ is the volume. Now we take:

$$
f_{k}-f_{k}^{o}=-\frac{\partial f^{o}}{\partial \epsilon_{k}} \delta \epsilon_{k} \quad \delta \epsilon_{k}=-e \vec{E} \cdot \vec{v}_{k} \tau(k),
$$

which defines the relaxation time $\tau(k)$, so that, according to Ohm's law, we get:

$$
\sigma=-\frac{2 e^{2}}{3 \mathcal{V}} \sum_{k} \vec{v}_{k} \cdot \vec{v}_{k} \tau(k) \frac{\partial f^{o}}{\partial \epsilon_{k}} .
$$

In th case of $s$ - wave scattering a spherical wave is diffracted from the impurity with maximum amplitude; this increases the flux propagating backward. Assuming that quantities depend on $\epsilon$ only and are mostly evaluated at the Fermi level we have $(T \sim 0)$ : 


$$
\sigma=-\frac{2 e^{2}}{3 \mathcal{V}} \nu(0) v_{F}^{2} \int d \epsilon \tau(\epsilon, T) \frac{\partial f^{o}}{\partial \epsilon}
$$

In this way we recover the simple microscopic formula for Ohm's conductivity, first derived by Drude in 1900, $\sigma=n e^{2} \tau / m$, where $\tau$ is an average relaxation time. The latter can be defined in terms of the mean free path between two scattering events $l=v_{F} \tau$ or distance between impurities. This formula is valid in the limit $k_{F} l>>1$ which we assume to be the case. In such a limit no localization effects occur and one can show that it is $d \rho / d T>0$ always, which is violated in the case of Kondo conductivity in diluted alloys.

In fact, for the large $U$ Anderson model, the explicit dependence of the transport time on temperature at the Fermi energy has to be taken into account separately. In addition, the Sommerfeld expansion can be used at finite temperatures [10]:

$$
\int d \epsilon\left(-\frac{\partial f(\epsilon)}{\partial \epsilon}\right) \tau(\epsilon, T)=\tau(\mu, T)+\left.\frac{\pi^{2}}{6}\left(k_{B} T\right)^{2} \frac{d^{2} \tau}{d \epsilon^{2}}\right|_{\epsilon=\mu}=\tau(\mu, 0)\left[1+\frac{\pi^{2}}{16 T_{K}^{2}}\left(\frac{1}{2} \pi^{2} T^{2}+\frac{\pi^{2}}{6} 3 T^{2}\right)\right] .
$$

The main ingredients to derive this formula are the Fermi liquid nature of the zero temperature GS and the Taylor expansion of the temperature dependence of the relaxation time. The prefactors have been specialized to the case of the symmetric Anderson model (so that the lifetime of the Kondo resonance is $\Gamma=4 k_{B} T_{K} / \pi$ ).

It follows that, in diluted alloys, the resistivity takes a maximum at $T=0$ :

$$
\frac{\rho(T)}{\rho(0)}=1-\frac{\pi^{4} T^{2}}{16 T_{K}^{2}}
$$

The extra correction on the r.h.s. of eq. (80) arises from inelastic scattering at finite temperature, as shown by Noziéres [40], according to the following argument.

In an elastic resonant scattering process at $T=0$ is $-\Im m\left\{t^{0}\right\}=\frac{1}{\nu(0) \pi} \sin ^{2} \delta^{0}$. In a diffusive $s$-wave scattering at finite $T$, we have to consider the total relaxation rate, defined as:

$$
\frac{1}{\tau(\epsilon)}=\sum_{k^{\prime}} w_{k k^{\prime}}\left(1-k \cdot k^{\prime}\right)=\frac{2 \pi}{\hbar} N_{i} \nu(0) \int d \epsilon_{k^{\prime}} \delta\left(\epsilon_{k}-\epsilon_{k^{\prime}}\right)|<k| t\left|k^{\prime}>\right|^{2}
$$

( $s$-wave scattering implies that the correction $k \cdot k^{\prime}$ averages to zero). The inelasticity can be accounted for by defining an effective elastic $S$-matrix which is now no longer unitary and an effective phase shift which is different from the one at $T=0$.

The full $S$-matrix, instead, is of course unitary: $1=\sum_{\beta}\left|S_{\alpha \beta}\right|^{2} \equiv \sum_{\beta}\left[\delta_{\alpha \beta}-2 \pi i \nu(0) t_{\alpha \beta} \delta\left(\epsilon_{\alpha}-\epsilon_{\beta}\right)\right]\left[\delta_{\alpha \beta}+\right.$ $\left.2 \pi i \nu(0) t_{\alpha \beta}^{*} \delta\left(\epsilon_{\alpha}-\epsilon_{\beta}\right)\right]=\sum_{\beta} \delta_{\alpha \beta}+\sum_{\beta} 4 \pi^{2}(\nu(0))^{2}\left|t_{\alpha \beta}\right|^{2} \delta\left(\epsilon_{\alpha}-\epsilon_{\beta}\right)$. Now, we separate the elastic channels $\beta=\alpha$ from the inelastic ones on the r.h.s. and we introduce the inelastic cross-section $W_{\alpha}^{\text {in }}=2 \pi \sum_{\beta \neq \alpha} \nu(0)\left|t_{\alpha \beta}\right|^{2} \delta\left(\epsilon_{\alpha}-\epsilon_{\beta}\right)$; hence, from unitarity it follows that $1+4 \pi^{2}(\nu(0))^{2}\left|t_{\alpha \alpha}\right|^{2}+2 \pi \nu(0) W_{\alpha}^{i n}=1$. Thus, we can define an effective phase shift (which we call $\delta$ again) in presence of inelastic scattering by introducing an effective "elastic" $S$ matrix:

$$
1-2 \pi i \nu(0) t_{\alpha \alpha}=\left(1-2 \pi \nu(0) W_{\alpha}^{i n}\right)^{\frac{1}{2}} e^{2 i \delta},
$$

where the square root can be expanded.

According to the optical theorem, we can write the total transmission probability as:

$$
W_{\alpha}=-2 \Im m\left\{t_{\alpha \alpha}\right\}=W_{\alpha}^{i n} \cos 2 \delta+\frac{(1-\cos 2 \delta)}{\nu(0) \pi} .
$$

Now, let us notice that, because $\delta \sim \pi / 2$, we have $\cos 2 \delta<0$. Therefore, for each channel $\alpha$ the quantity $W_{\alpha}$ is always smaller than the elastic case value: $2 \sin ^{2} \delta /(\nu(0) \pi)$. But one can thermally average over the $\alpha$ channels; according to the usual phase space argument which is invoked when scattering occurs close to the Fermi surface at very low $T$, we obtain an average $\overline{W^{i n}}$ proportional to $T^{2}: \overline{W^{i n}} \sim A T^{2}$. This gives rise to the first term in the expansion of eq. (80). The second term can be viewed as contributing to the Sommerfeld expansion with $\delta(\epsilon)=\frac{1}{2} \pi-a \epsilon$ from which it follows that $\sin ^{2} \delta \sim 1-a^{2} \epsilon^{2}$.

In the case of resonant tunneling across the dot, the conductance is given by eq. (20). Using the result of eq. (83) directly one obtains that conductance has a maximum at zero temperature and first corrections in temperature are again of $\mathcal{O}\left(T^{2}\right)$ (see eq. (49)). 


\section{Acknowledgements}

This manuscript is the revised and updated version of the lectures delivered by one of us (A. T.) at INFN Laboratories in Frascati (Italy) during the school "Nanotubes \& Nanostructures" (October 18-27, 2001). A. T. wishes to thank S. Bellucci and M. De Crescenzi for the invitation in such a warm and stimulating atmosphere. A. Naddeo was supported by a CNR fellowship while this work was done.

[1] L. I. Glazman and M. E. Raikh, Pis'ma Zh. Eksp. Teor. Fiz. 47, 378 (1988) [JETP Lett. 47, 452 (1988)].

[2] I. L. Aleiner, P. W. Brouwer and L. I. Glazman, Phys. Rep. 358, 309 (2002).

[3] D. Goldhaber-Gordon, H. Shtrikman, D. Mahalu, D. Abusch-Magder, U. Meirav and M. A. Kastner, Nature 391, 156 (1998); S. M.Cronenwett, T. H. Oosterkamp and L. P. Kouwenhoven, Science 281, 540 (1998); J. Schmid, J. Weis, K. Eberl and K. v.Klitzing, Physica B256-258,182 (1998); S. Sasaki, S. De Franceschi, J. M. Elzerman, W. G. van der Wiel, M. Eto, S. Tarucha and L. P. Kouwenhoven, Nature 405, 764 (2000).

[4] J. Kondo, Prog. Theor. Phys. 32, 37 (1964).

[5] A. C. Hewson, "The Kondo Effect to Heavy Fermions", Cambridge University Press, Cambridge (1993).

[6] K. G. Wilson, Rev. Mod. Phys. 47, 773 (1975); H. R. Krishnamurthy, J. W. Wilkins and K. G. Wilson, Phys. Rev. B21, 1003 (1980).

[7] A. M. Tsvelick and P. B. Wiegmann, Advances in Physics 32, 453 (1983).

[8] I. Affleck and A. W. W. Ludwig, Phys. Rev. B48, 7297 (1993); I. Affleck and A. W. W. Ludwig, Nucl. Phys. B352, 849 (1991); I. Affleck and A. W. W. Ludwig, Nucl. Phys. B360, 641 (1991); I. Affleck and A. W. W. Ludwig, Phys. Rev. Lett. 67, 161 (1991); I. Affleck, Acta Phys. Polon. B 26, 1869 (1995).

[9] N. E. Bickers, Rev. Mod. Phys. 59, 845 (1987).

[10] T. A. Costi, A. C. Hewson and V. Zlatic, J. Phys. Cond. Matt. 6, 2519 (1994); T. A. Costi and A. C. Hewson, Phil. Mag. $B$ 65, 1165 (1992).

[11] T. A. Costi, Phys. Rev. B64, 24130(R) (2001); T. A. Costi, Phys. Rev. Lett. 85, 1504 (2000).

[12] O. Sakai and Y. Shimizu, J. Phys. Soc. Jpn. 61, 2333 (1992); O. Sakai, S. Suzuki and Y. Shimizu, J. Physica B206-207, 141 (1995); W. Izumida, O. Sakai and Y. Shimizu, J. Phys. Soc. Jpn. 66, 717 (1997); W. Izumida, O. Sakai and Y. Shimizu, J. Phys. Soc. Jpn. 67, 2444 (1998).

[13] T. K. Ng, Phys. Rev. Lett. 61, 1768 (1988).

[14] S. Hershfield, J. H. Davies and J. W. Wilkins, Phys. Rev. Lett. 67, 3270 (1991).

[15] Y. Meir, N. S. Wingreen and P. A. Lee, Phys. Rev. Lett. 70, 2601 (1993); Y. Meir and N. S. Wingreen, Phys. Rev. Lett. 68, $2512(1992)$.

[16] J. Koenig, J. Schmidt, H. Schoeller and G. Schon, Phys. Rev. B54, 16820 (1996); Czech. J. Phys. 46 S 4, 2399 (1996).

[17] Y. Meir and N. S. Wingreen, Phys. Rev. B49, 11040 (1994).

[18] M. H. Hettler, J. Kroha and S. Hershfield, Phys. Rev. B58, 5649 (1998).

[19] H. Schoeller and G. Schon, Phys. Rev. B50, 18436 (1994); H. Schoeller, in "Mesoscopic electron transport", L. Sohn, L. P. Kouwenhoven and G. Schön eds., NATO ASI Series E 345,105, Kluwer, Dordrecht, Netherlands (1997), pg. 291-330; H. Schoeller, Lect. Notes Phys. 544, 137 (2000).

[20] H. Schoeller and J. Koenig, Phys. Rev. Lett. 84, 3686 (2000); J. Koenig and H. Schoeller, Phys. Rev. Lett. 81, 3511 (1998).

[21] P. W. Anderson, G. Yuval and D. R. Hamann, Phys. Rev. B1, 4464 (1970); D. R. Hamann, Phys. Rev. B2, 1373 (1970).

[22] L. I. Glazman and M. Pustilnik, cond-mat/0302159, to appear in Proceedings of the NATO ASI "New Directions in Mesoscopic Physics", Erice (2002).

[23] D. Giuliano, B. Jouault and A.Tagliacozzo, Phys. Rev. B63,125318 (2001); D. Giuliano, B. Jouault and A. Tagliacozzo, in "Macroscopic Quantum Coherence and Quantum Computing", D. V. Averin et al. eds., Kluwer Academic/Plenum Publishers, New York (2001), pg. 325.

[24] D. Giuliano, B. Jouault and A. Tagliacozzo, Europhys. Lett. 58(3), 401 (2002).

[25] Y. Oreg and D. Goldhaber-Gordon, Phys. Rev. Lett. 90, 136602 (2003).

[26] L. I. Glazman and M. Pustilnik, Phys. Rev. Lett. 91, 066405 (2003).

[27] L. P. Kouwenhoven et al., in "Mesoscopic electron transport", L. Sohn, L. P. Kouwenhoven and G. Schön eds., NATO ASI Series E 345,105, Kluwer, Dordrecht, Netherlands (1997).

[28] B. Jouault, G. Santoro and A. Tagliacozzo, Phys. Rev. B61, 10242 (2000).

[29] M. A. Kastner and D. Goldhaber-Gordon, Solid State Comm. 119, 245 (2001).

[30] T. K. Ng and P. A. Lee, Phys. Rev. Lett. 61, 1768 (1988).

[31] A. P. Jauho, N. S. Wingreen and Y. Meir, Phys. Rev. B50, 5528 (1994).

[32] L. V. Keldysh, Zh. Eksp. Teor. Fiz. 47, 1515 (1965) [Sov. Phys. JETP 20, 1018 (1965)]; G. D. Mahan, "Many-Particle Physics", Plenum, New York (1990), 2nd ed.. 
[33] A. Tagliacozzo and E. Tosatti, Physica Scripta 38, 301 (1988).

[34] B. Nienhuis, "Coulomb Gas Formulation of Two-dimensional Phase Transitions", C. Domb and J. Lebowitz eds., Academic Press (1987).

[35] D. Giuliano and A. Tagliacozzo, Phys. Rev. Lett. 84, 4677 (2000).

[36] J. Kondo, J. Appl. Phys. 37, 1177 (1966).

[37] W. G. van der Wiel, S. De Franceschi, T. Fujisawa, J. M. Elzerman, S. Tarucha and L. P. Kouwenhoven, Science 289, 2105 (2000).

[38] J. R. Schrieffer and P. A. Wolff, Phys. Rev. 149, 491 (1966).

[39] P. W. Anderson, J. Phys. C3, 2436 (1970).

[40] P. Noziéres, J. Low Temp. Phys. 17, 31 (1974).

[41] P. Nozierés and A. Blandin, J. Phys. Paris 41, 193 (1980).

[42] M. Fabrizio, A. O. Gogolin and P. Noziéres, Phys. Rev. B51, 16088 (1995).

[43] N. Andrei, Phys.Lett A87, 299 (1982); N. Andrei, K. Furuya and J. H. Lowenstein, Rev.Mod.Phys. 55, 331 (1983).

[44] Yu. N. Ovchinnikov and A. M. Dyugaev, JETP Lett. 70, 111 (1999); JETP Lett. 88, 696 (1999).

[45] S. Tarucha, D. G. Hausting, T. Honda, R. J. van der Hage and L. P. Kouwenhoven, Phys. Rev.Lett. 77, 3613 (1996).

[46] M. Eto and Y. Nazarov, Phys. Rev.Lett. 85, 1306 (2000).

[47] M. Pustilnik, L. I. Glazman, D. H. Cobden and L. P. Kouwenhoven, Lect. Notes Phys. 579, 3 (2001); M. Pustilnik and L. I. Glazman, Phys. Rev.Lett. 85, 2993 (2001); Phys. Rev. B64, 045328 (2001).

[48] I. Affleck, A. W. W. Ludwig and B. A. Jones Phys. Rev. B52, 9528 (1995).

[49] M. Wagner, U. Merkt and A. V. Chaplik, Phys. Rev. B45, 1951 (1992); P. Lucignano, B. Jouault, and A. Tagliacozzo, Phys. Rev. B69, 045314 (2004).

[50] D. C. Ralph, A. W. W. Ludwig, J. von Delft and R. A. Buhrman, Phys. Rev. Lett. 72,1064 (1994); S. K. Upadhyay, R. N. Louie and R. A. Buhrman, Phys. Rev. B56, 12033 (1997).

[51] G. Zaránd and A. Zawadowsky, Phys. Rev. Lett. 72, 542 (1994); Phys. Rev. B50, 932 (1994); D. L. Cox and A. Zawadowsky, Adv. Phys. 47, 599 (1998).

[52] I. L. Aleiner, B. L. Altshuler and Y. M. Galperin, cond-mat 0102513.

[53] M. H. Hettler, J. Kroha and S. Hershfield, Phys. Rev. Lett. 73,1967 (1994).

[54] J. M. Luttinger, Phys. Rev. 121, 942 (1960).

[55] A. L. Fetter and J. D. Walecka, "Quantum Theory of Many-Particle Systems", McGraw-Hill Editions (1981).

[56] K. Vladar and A. Zawadowsky, Phys. Rev. B28, 1564, 1582, 1596 (1983).

[57] A. Muramatsu and F. Guinea, Phys. Rev. Lett. 57, 2337 (1986).

[58] V. J. Emery and S. Kivelson, Phys. Rev. B46, 10812 (1992); Phys. Rev. Lett. 71, 3701 (1993).

[59] J. M. Maldacena and A. W. W. Ludwig, Nucl. Phys. B506, 565 (1997).

[60] P. Coleman, L. Ioffe and A. M. Tsvelick, Phys. Rev. B52, 6611 (1995).

[61] J. von Delft, G. Zaránd and M. Fabrizio, Phys. Rev. Lett. 81, 196 (1998).

[62] N. W. Ashcroft and N. D. Mermin, "Solid State Physics", Holt-Saunders International Editions, Tokyo (1976).

\section{Figure Captions}

- Figure 1: Schematic drawing for a dot and the contacts in a vertical setup; possibly a magnetic field is applied along the axis.

- Figure 2: Differential conductance on a gray scale as a function of both $V_{g}$ and $V_{d s}$; the Kondo effect shows up near $V_{d s}=0[29]$.

- Figure 3: Renormalization-group flow diagram for small $J[21]$.

- Figure 4: Qualitative renormalization-group flow diagram for the anisotropic two channel Kondo problem; the non trivial fixed point corresponds to $J_{1}=J_{2}=J_{*}$ (channel symmetry) [42].

- Figure 5: Plot of the universal function $f(x)$ vs $x=T / T_{K}[11]$.

- Figure 6: Sketch of the conductance across the dot as a function of the temperature $T$ and the gate voltage $V_{g}$ $[24]$.

- Figure 7: Second-order diagrams $\mathcal{D}_{1}$ and $\mathcal{D}_{2}$. Conduction fermions are represented as full lines, while dashed lines represent propagation of $d$-fermions (dot's states).

- Figure 8: Third-order vertex renormalization: the first one $(P)$ is a "parquet-type" diagram. Its contribution is accounted for in the integration of the second-order RG equation. The second one (NP) is a "non parquet" diagram. It provides an additional third-order contribution to the $\beta$-function. 

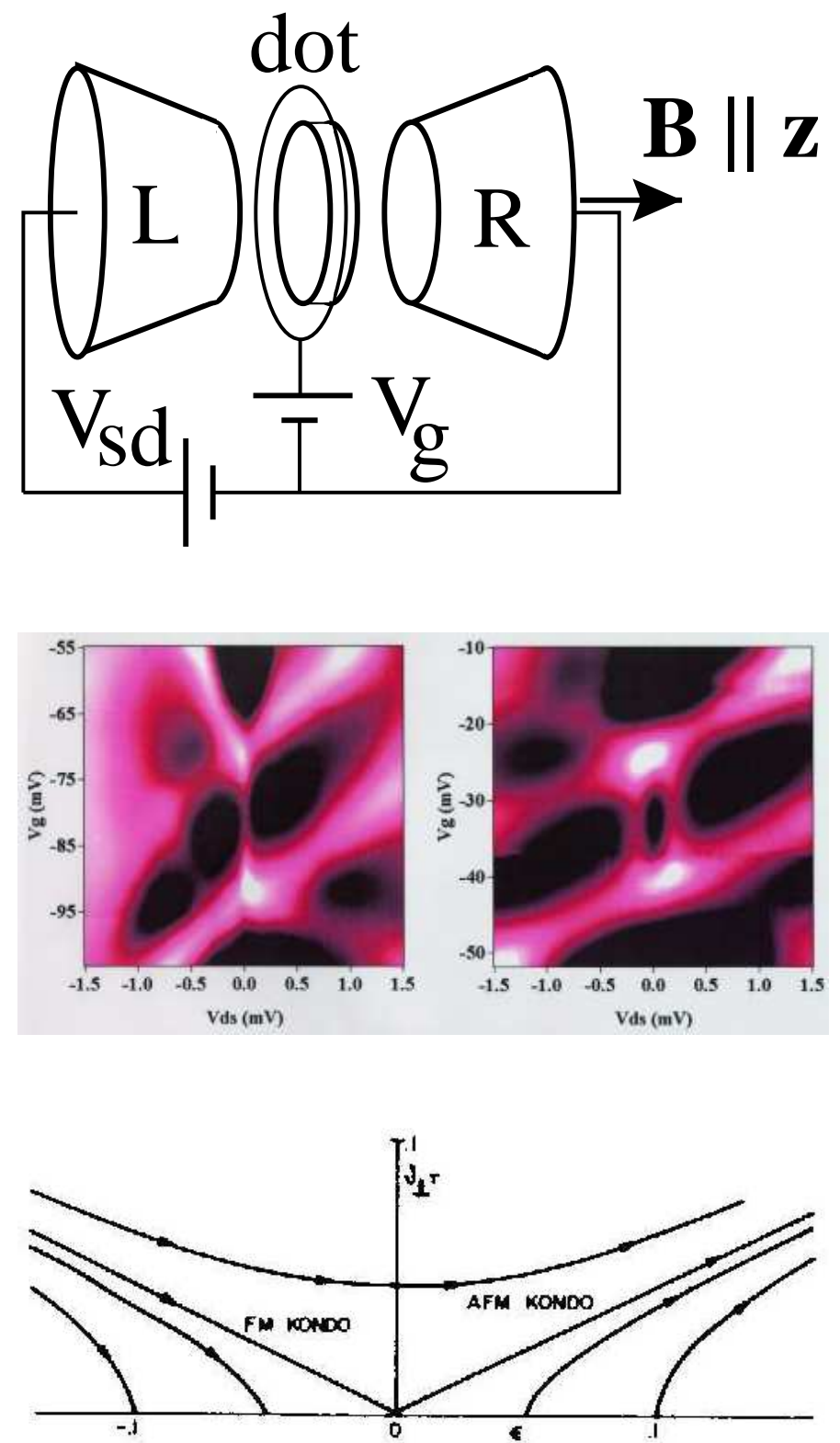

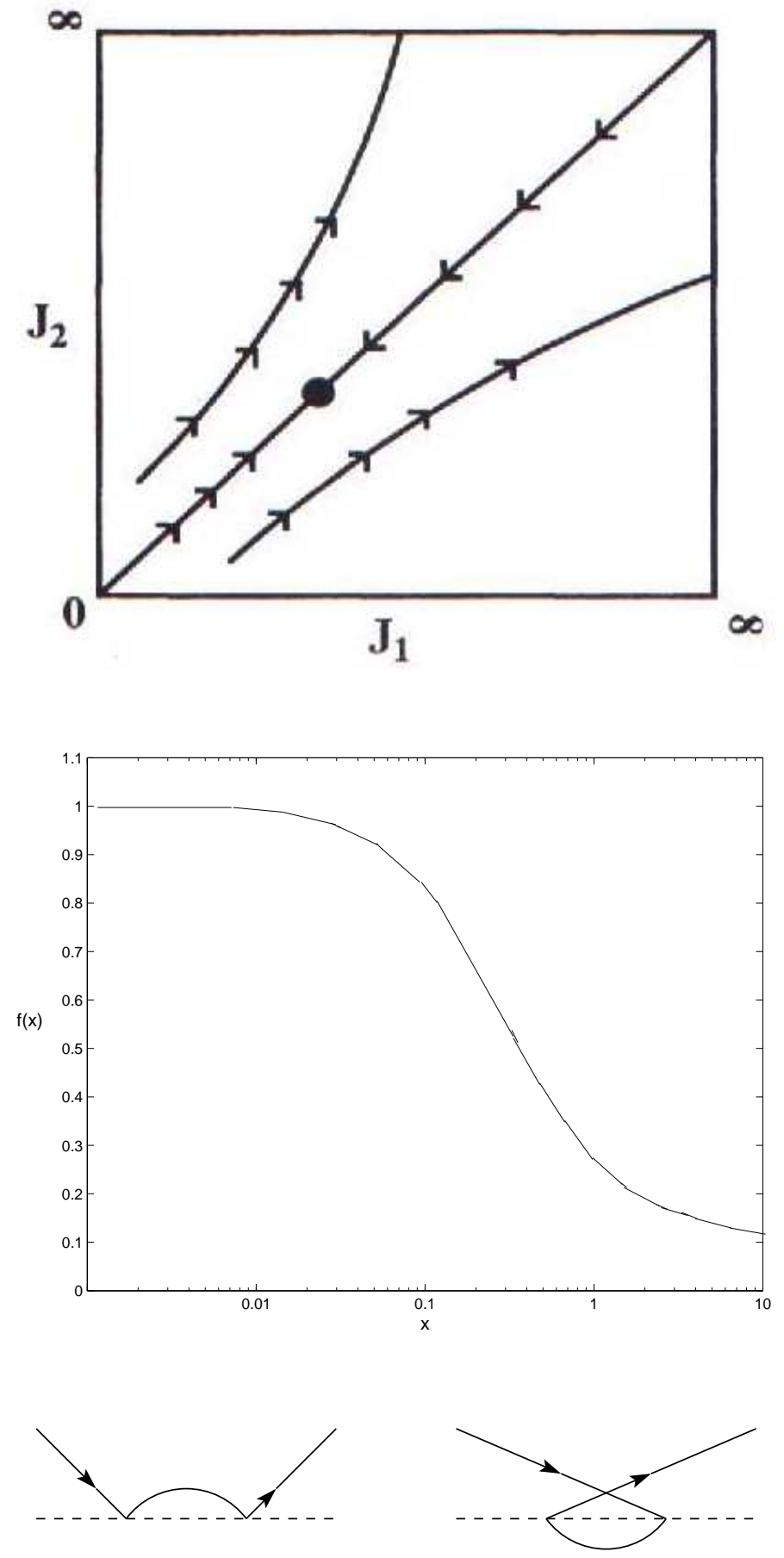

D

$D_{2}$ 

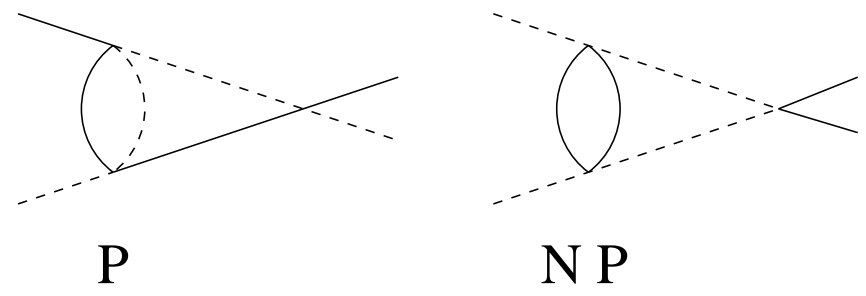